\title{
Non-linear MHD simulations of sawteeth and their control by current and power depositions
}

\author{
O. Février ${ }^{1,2}$, T. Nicolas ${ }^{2}$, P. Maget ${ }^{1}$, J-H. Ahn ${ }^{1,3}$, X. Garbet ${ }^{1}$, \\ H. Lütjens ${ }^{4}$ \\ ${ }^{1}$ CEA, IRFM, F-13108 Saint Paul-lez-Durance, France. \\ ${ }^{2}$ École Polytechnique Fédérale de Lausanne (EPFL), Swiss Plasma Center, Station \\ 13, CH-1015 Lausanne, Switzerland. \\ ${ }^{3}$ Department of Applied Physics and Applied Mathematics, Columbia University, \\ New York, NY 10027, USA. \\ ${ }^{4}$ Centre de Physique Théorique, École Polytechnique, CNRS, France. \\ E-mail: olivier.fevrier@epfl.ch
}

\begin{abstract}
Sawteeth in tokamak plasmas correspond to periodic relaxations of the temperature and density in the central region of the plasma, caused by the internal kink mode. They play an important role in core confinement and impurity transport in the central region of a tokamak discharge, and they can trigger secondary instabilities. Being able to control their dynamics is therefore important. In this article, we explore by means of MHD simulations the control of sawteeth, relying on current or power deposition in the vicinity of the $q=1$ surface. To do so, simulations with the full-MHD code XTOR-2F [1], which features power and current source terms mimicking the effects of ECCD and ECRH, are performed. Simulations show that a deposition of current inside the inversion radius leads to an increase of the sawtooth frequency whereas a deposition near or outside the inversion radius leads to a decrease of the sawtooth frequency, in agreement with theoretical and experimental predictions. For power depositions, the situation appears however to be more complex. The modification of the sawtooth shape in presence of additional heating or current drive is also investigated. The existence of a critical shear setting the condition of the sawtooth crash is found to apply, although the inter-crash dynamics is often too complex and MHD-dependent to be summarized as a periodic instantaneous redistribution of current and energy, as assumed in the analytical models. Finally, we briefly explore possible scenarios for the triggering of magnetic islands, and show how controlling the sawteeth can help to prevent the triggering of tearing instabilities.
\end{abstract}

Submitted to: Nucl. Fusion 
Non-linear MHD simulations of sawteeth and their control by current and power depositions 2

\section{Introduction}

The sawtooth instability in tokamak plasmas manifests itself as a periodic relaxation of the temperature and density in the central region of the plasma. These relaxations are caused by the $(m=1, n=1)$ internal kink mode [2,3,4]. A sawtooth is characterized by a ramp phase and a crash phase. During the ramp phase, the core temperature and pressure steadily rise during a timescale typically ranging from few milliseconds to several seconds, depending on plasma parameters [5]. This phase is followed by a "crash", during which temperature and pressure collapse and flatten in the central region [6], the hot core being expelled, in a characteristic timescale of the order of a hundred of microseconds $[7,8,9]$. This leads to a drop of the confinement, and therefore to a reduction of the device performances. Sawteeth are also known to play a key role in the dynamics of the impurities in the core region, and thus might be useful to prevent impurity accumulation in the core region [10, 11, 12, 13]. Unfortunately, sawtooth crashes are also susceptible to lead to the onset of Neoclassical Tearing Modes (NTMs) $[14,15,16,17,18,19]$, which can also in turn lower the confinement or lock [20] and lead to disruptions [21]. The contribution of fast particles, such as the fusion-born $\alpha$ particles or those induced by NBI or ICRH, can lead to the stabilization of the internal kink mode $[22,23]$, and therefore to the increase of the sawtooth period, which might be problematic as it has been shown experimentally that longer sawtooth lead to higher chance of triggering a NTM $[15,16]$. All these reasons call for the investigation of the possibility to influence the sawtooth period.

Two main methods have been explored [24]. In the first one, the population of fast particles in the core plasma is modified to alter the stability of the internal kink mode $[25,22,26,23,27]$ and ultimately the sawtooth period. This has been achieved experimentally using either NBI $[28,29,30]$ or ICRH [31]. The second method relies on the use of external current-drive or heating to affect the sawtooth dynamics. The behavior of the sawteeth can be understood in the framework of critical shear-model, where the sawteeth are triggered when a critical value of the shear on $q=1$ is reached $[32,33]$. By changing the $q$ profile near $q=1$, it is therefore possible to modify the stability of the internal kink mode, and trigger or stabilize the sawteeth. This can be achieved using external current-drive [34, 35, 14, 36, 37, 38, 39, 40, 41, 42], or by modifying the temperature profile through localized heating so as to change the plasma resistivity and therefore the inductive current profile[43, 14, 44, 45]. To actively tailor the sawtooth period or to choose the moment at which the crash happens, control schemes relying on the modulation of the $\mathrm{RF}$ power have been developed, such as sawtooth locking [46] or sawtooth pacing [47]. In ITER, the use of a $20 \mathrm{MW}$ EC to control the sawteeth is foreseen [48].

In this article, we investigate the impact of continuous current or power depositions on the dynamics and shape of sawteeth from the simulation point of view using the full-MHD code XTOR-2F [1, 49], which has been upgraded to include an ECCD-like source term [50]. This allows us to describe in a self-consistent way the evolution of the 
sawteeth and their response to the current or power depositions. In section 2, we present the MHD equilibrium that is used in the simulations, as well as the MHD model used in XTOR-2F. The question of the Ohm's law formulation, which appears critical to ensure the correct description of the different phenomena at play, is addressed in section 3. We then investigate in section 4 the modification of the sawtooth period by current or power depositions. The simulations show that the deposition of current inside the inversion radius leads to an increase of the sawtooth frequency whereas a deposition near or outside the inversion radius leads to a decrease of the sawtooth frequency, in agreement with theoretical predictions based on a critical shear model and experiments. For power depositions, the picture is more complex. In particular, it appears that if the total injected power is too low, then the sawtooth period is increased for all deposition locations. We further observe that for a current deposition inside the inversion radius, the sawtooth crashes are not brutal anymore, but feature long-lived rotation of the hot core before its expulsion. Similar modifications of the sawtooth shape are observed for power depositions. We then explore the role of the ohmic contribution of the plasma current on the sawtooth dynamics. The fraction of ohmic current in the total plasma current appears to be also a key parameter to explain the results obtained with power depositions. In section 5, the simulation results obtained with XTOR are compared with a $1 \mathrm{D}$ critical shear model. The concept of a critical shear as a criterion for the onset of the sawtooth crash is found to be consistent with numerical simulations, although existing expressions in the literature $[32,33]$ do not apply to the situation considered, where the density gradient is vanishing. In the case of pure current drive, a critical shear model is shown to give a reasonable prediction of the sawtooth period dependence on the current deposition. But the agreement is poor regarding the case of pure heating. More generally, the dynamics of the sawtooth, and therefore the determination of the sawtooth period, is too complex in many situations to be summarized as a periodic instantaneous redistribution of current and energy, assumed in the analytical models. The incidence of such complexity was evidenced in the modeling of partial sawtooth reconnection [51], and it also impacts the triggering of other MHD modes that are sensitive to the detailed shape of the equilibrium in the course of the sawtooth cycle, like electron fishbone modes [52] or other fast particle driven modes [53]. In section 6, we present some results on the modification of the linear stability of tearing modes during the sawtooth crash, and investigate the effect of current deposition on the possible triggering of a metastable island.

\section{Equilibrium and MHD model}

We use a circular cross-section magnetic equilibrium computed using the GradShafranov code CHEASE [54]. The inverse aspect ratio is $\epsilon=0.37$ and the major radius $R_{0}=1.0 \mathrm{~m}$. The pressure and safety factor $(q)$ profiles associated with this equilibrium are plotted in figure 1 . The central magnetic field is $B_{0}=1$ T. $q=1$ is located at $\rho=\sqrt{\psi}=0.392, \rho$ being the square root of the normalized poloidal flux, and 
Non-linear MHD simulations of sawteeth and their control by current and power depositions4

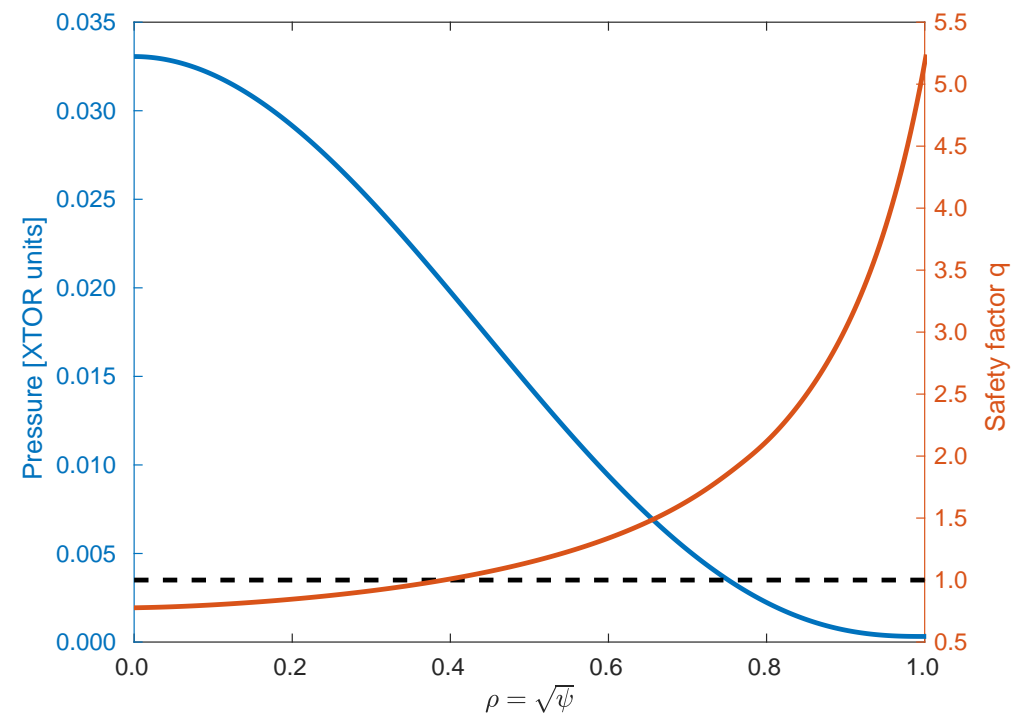

Figure 1. Equilibrium pressure and safety factor profiles for the equilibrium considered in this paper.

playing the role of a minor radius coordinate. This equilibrium is similar to the one used in Refs. [55, 56], although the current profile has been slightly altered in the vicinity of $q=2$ in order to increase the linear stability of the $(2,1)$ mode. This equilibrium is then used as an input for the two-fluid, resistive model of the 3D full-MHD code XTOR-2F $[1,49]$, which has been upgraded to include a current source term mimicking ECCD in the Ohm's law, thus allowing to study the control of MHD instabilities [50]. The equations solved are, in XTOR normalized units [1]:

$$
\begin{aligned}
& \partial_{t} N+\nabla \cdot(N \mathbf{V})+\frac{\nabla p_{i}}{e_{i}} \cdot \mathbf{K}=\nabla \cdot\left(D_{\perp} \nabla N\right)+S_{N} \\
& N m_{i}\left[\partial_{t} \mathbf{V}+(\mathbf{V} \cdot \nabla) \mathbf{V}+\left(\mathbf{V}_{i}^{*} \cdot \nabla \mathbf{V}_{\perp}\right)\right] \\
& =\mathbf{J} \times \mathbf{B}-\nabla p+\nu \nabla^{2}\left(\mathbf{V}+\mathbf{V}_{i}^{*}\right) \\
& \partial_{t} p+\mathbf{V} \cdot \nabla p+\Gamma p \nabla \cdot \mathbf{V}+ \\
& \frac{\Gamma}{e_{i}}\left(T \nabla p_{i}+p_{i} \nabla T_{i}+p_{e} \nabla T_{e}\right) \cdot \mathbf{K} \\
& =(\Gamma-1) \nabla \cdot \mathbf{q}^{\chi}+S_{H}+S_{R F} \\
& \partial_{t} \mathbf{B}-\nabla \times\left[\eta\left(\mathbf{J}-\mathbf{J}_{\mathbf{C D}}-\mathbf{J}_{\mathbf{R F}}\right)\right] \\
& =\nabla \times(\mathbf{V} \times \mathbf{B})+\nabla \times\left(\frac{\nabla_{\|} p_{e}}{N e}\right) \\
& \frac{\partial J_{R F}}{\partial t}+\nu_{f}\left(J_{R F}-J_{R F}^{s}\right) \\
& =\chi_{\perp}^{R F} \nabla^{2} J_{R F}+\chi_{\|}^{R F} \nabla_{\|}^{2} J_{R F} \\
& \frac{\partial S_{R F}}{\partial t}+\nu_{f}\left(S_{R F}-S_{R F}^{s}\right) \\
& =\chi_{\perp}^{R F} \nabla^{2} S_{R F}+\chi_{\|}^{R F} \nabla_{\|}^{2} S_{R F}
\end{aligned}
$$


In these equations, the indexes $i$ and $e$ denote the ion and electron populations. $\mathbf{V} \equiv \mathbf{E} \times \mathbf{B} / B^{2}+\mathbf{V}_{\|, i}$ is the plasma fluid velocity, while $\mathbf{V}_{i}^{*} \equiv \mathbf{B} \times \nabla p_{i} /\left(N_{i} e_{i} B^{2}\right)$ is the ion diamagnetic velocity. $\mathbf{K}=\nabla \times \frac{\mathbf{B}}{B^{2}}$ is the curvature. $p=p_{e}+p_{i}$ is the total pressure, $N=N_{e}=N_{i}$ is the plasma density, quasi-neutrality being assumed, and $m_{i}$ is the ion mass. The ratio of ion to electron temperatures is $\tau \equiv T_{i} / T_{e}=1$. In equation $1, S_{N}$ is a particles source, the role of which is to restore the equilibrium density profile. In equation $3, S_{H}=-\nabla \cdot\left(\rho^{e q} \chi_{\perp}^{e q} \nabla_{\perp} T^{e q}\right)$ (where the superscript "eq" denotes the initial equilibrium value of the quantity) is a pressure source term, the role of which is to restore the equilibrium pressure profile. $\Gamma=5 / 3$ is the ratio of specific heat. The heat flux is defined as $\mathbf{q}^{\chi}=-N \chi_{\|} \nabla_{\|} T-N \chi_{\perp} \nabla_{\perp} T$, where $T=p / N$. $\chi_{\|}$ and $\chi_{\perp}$ are diffusion coefficients, the former accounting for the parallel transport, while the latter accounts for the perpendicular transport, modeling both the collisional and turbulent processes. $\chi_{\perp}$ is chosen such that $\chi_{\perp} / \eta=45$, and $\chi_{\|}$is chosen such that $\chi_{\|} / \chi_{\perp} \approx 3 \times 10^{6} . D_{\perp}$ is the perpendicular particle diffusion coefficient. $\eta$, the plasma resistivity, varies in time with the plasma temperature, following the Spitzer's resistivity law $\eta \propto T^{-3 / 2}$. The Lundquist number is $S=\tau_{R} / \tau_{A}=10^{7}$, where $\tau_{R}=\mu_{0} a^{2} / \eta$ is the resistive timescale and $\tau_{A}=R_{0} \sqrt{\mu_{0} N_{0} m_{i}} / B_{0}$ the Alfvén time. The magnetic Prandtl number is set to Prm $=\nu / \eta=50$. $\mathbf{J}_{\mathbf{C D}}$ is a current source intended to restore the equilibrium current profile, and its expression and role on the sawtooth dynamics will be presented in section 3. It is constant throughout the simulation. $\mathbf{J}_{\mathbf{R F}}=J_{R F} \mathbf{b}$, where $\mathbf{b}=\mathbf{B} / B$, models a RF current source term. The model retained for equations 5 and 6 is presented in detail in Ref. [50]. XTOR normalizations are detailed in Ref [1]. The mesh used in the simulations is $\left(N_{r}, N_{\theta}, N_{\phi}\right)=(256,32,12)$, where $N_{\rho}, N_{\theta}, N_{\phi}$ are the number of grid points in $\rho, \theta, \phi$ directions. A total of 4 toroidal modes $(n=0, \ldots, 3)$ are described, each of them including $n+8$ poloidal modes $(m=0, \ldots, n+7)$. The question of the resolution required for XTOR simulations of sawteeth crashes has been addressed in Refs $[1,55,51]$. Unless stated otherwise, all results presented in this paper have be obtained with XTOR simulations using equations 1-6.

\section{Formulation of the current source term in Ohm's law}

In this section, we focus on the Ohm's law (equation 4), and on the different source terms it can contain. The Ohm's law can be written as

$$
\mathbf{E}+\mathbf{V} \times \mathbf{B}=\eta\left(\mathbf{J}-\mathbf{J}_{\mathbf{C D}}-\mathbf{J}_{\mathbf{R F}}\right)-\left(\frac{\nabla_{\|} p_{e}}{N e}\right) \mathbf{b}
$$

where $\mathbf{J}$ is the plasma current, $\mathbf{J}_{\mathbf{C D}}$ a non-inductive contribution to the plasma current and $\mathbf{J}_{\mathbf{R F}}$ the non-inductive source term mimicking external current deposition for sawtooth or tearing modes control, introduced in Ref. [50]. $\eta$ is the plasma resistivity, specified as a local function of the temperature:

$$
\eta(\rho, \theta, \phi) \propto T(\rho, \theta, \phi)^{-\frac{3}{2}}
$$

In the following, we explain how $\mathbf{J}_{\mathbf{C D}}$ is crafted in our simulations so that the resistive term in the Ohm's law acts as a restoring force that tends to relax the plasma equilibrium 
Non-linear MHD simulations of sawteeth and their control by current and power depositions6

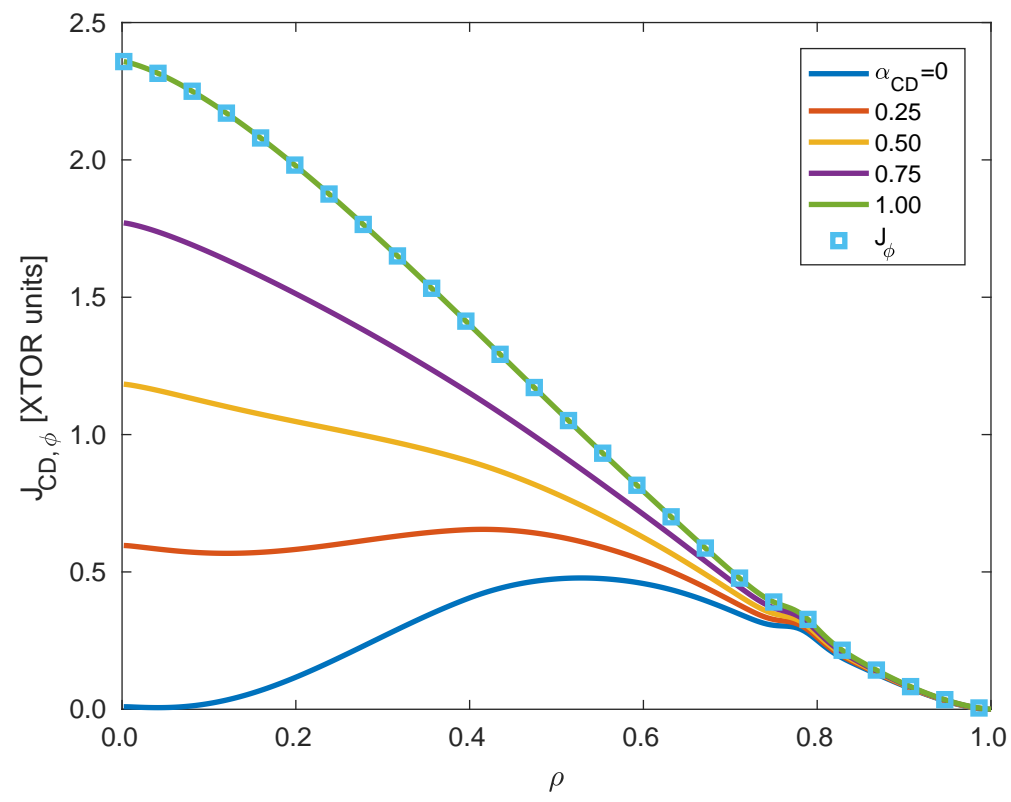

Figure 2. Profile of the $\mathbf{J}_{\mathbf{C D}}$ source term (equation 4) in an equatorial plane $(\theta=0$, $\phi=0$ ) for different values of the scaling parameter $\alpha_{C D}$. The squares indicate the profile of the toroidal component of the plasma current, $J_{\phi}$.

towards the initial, ideal equilibrium provided by CHEASE if the temperature profile remains unchanged. Denoting by $\eta_{0}^{e q}$ the value of $\eta$ at equilibrium on the magnetic axis, and assuming that at this point, the current on the magnetic axis is provided solely by the inductive mechanism, the electric field at the center of the plasma can be evaluated as $E_{0}=\eta_{0}^{e q} J_{0, \phi}^{e q}$, where $J_{0, \phi}^{e q}$ is the value of $J_{\phi}$ at equilibrium on the magnetic axis. One should note that defining the electric field from the quantities at the center of the plasma is a choice made for convenience, and a similar implementation could be achieved considering edge quantities. We then introduce the scaling parameter $\alpha_{C D}$, which allows us to control the contribution of the non-inductive current to the total plasma current, by introducing the electric field $\tilde{E}_{0}=\left(1-\alpha_{C D}\right) E_{0}$. We can now define $J_{C D}$ such that

$$
J_{C D}=J_{\phi}^{e q}-\frac{\tilde{E}_{0}}{\eta^{e q}}=J_{\phi}^{e q}-\frac{\left(1-\alpha_{C D}\right) E_{0}}{\eta^{e q}}
$$

In figure 2, we have plotted the profile of $J_{C D}$ in an equatorial plane $(\theta=0, \phi=0)$ for different values of the scaling parameter $\alpha_{C D}$. The boundary condition $E_{\phi}^{e d g e}=\tilde{E}_{0}$ is imposed. With this formulation, one can explore different regimes of current dynamics. In the case of $\alpha_{C D}=1, J_{C D}=J_{\phi}^{e q}$ and therefore the plasma current is hooked to the equilibrium current, so that the variations of the resistivity $\eta$ do not directly reflect on the current profile. $\eta$ however still plays a role in the dynamics itself, by influencing the timescale over which the current reaches its equilibrium value. For $\alpha_{C D} \neq 1$, since $J_{C D} \neq J_{\phi}^{e q}$, part of the current will be inductive and will diffuse so that $\eta\left(J_{\phi}^{e q}-J_{C D}\right)$ tends to $E_{\phi}^{e d g e} \neq 0$. In that case, changes of the resistivity will impact the value of the central current density. This is illustrated in the top panel of figure 3, where the 
Non-linear MHD simulations of sawteeth and their control by current and power depositions7
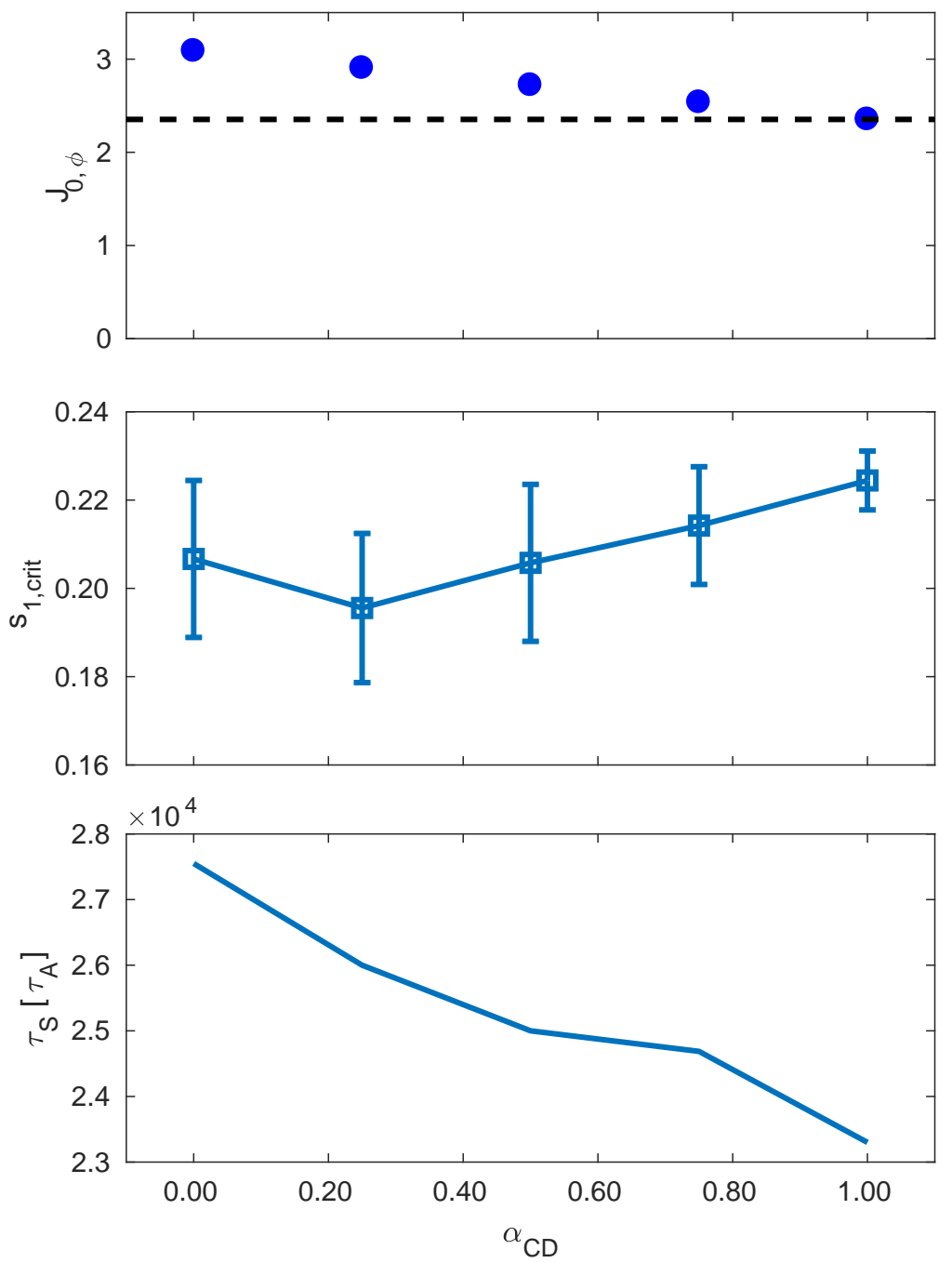

Figure 3. (top) Evolution of the central toroidal current density $J_{0, \phi}^{e q}$ plotted as a function of $\alpha_{C D}$, which represents the fraction of non-inductive current supplied at the center, when temperature is increased by a factor of 1.2 (the heat source term $S_{H}$ is increased by this factor during the simulation). The dashed line indicates the initial value of $J_{0, \phi}$. It should be noted that in this figure, a lower Lundquist number $\left(S=10^{5}\right)$ has been used to reduce the resistive time and thus the time required for the current diffusion. (middle) Evolution of the shear on $q=1$ at the time of the sawtooth crash $s_{1, \text { crit }}$ as a function of $\alpha_{C D}$. The error bars indicate the dispersion of the critical shears measured in the simulations. A dispersion of about $10 \%$ is observed. (bottom) Evolution of the sawtooth period for the equilibrium presented in section 2 for different values of $\alpha_{C D}$.

evolution of the central toroidal current density $J_{0, \phi}^{e q}$ is plotted as a function of $\alpha_{C D}$, which represents the fraction of non-inductive current supplied at the center, when temperature is increased by a factor of 1.2 (the heat source term $S_{H}$ is increased by this factor during the simulation). As expected, when $\alpha_{C D}=1$, the current density is hooked to its equilibrium value and the modification of resistivity induced by the heating is not reflected on the current density. On the contrary, for $\alpha_{C D} \neq 1$, one can 
see that the current density increases in response to the drop of resistivity caused by the heating. Increasing the fraction of ohmic current (that is, decreasing $\alpha_{C D}$ ) enhances this effect. From this analysis, it is also clear that simulations including external heating sources must be done with $\alpha_{C D} \neq 1$ in order to correctly reflect the impact of heating not only on the pressure profile, but also on the current profile. We stress out that, as shown in figure $2, \alpha_{C D}=0$ is not equal to purely ohmic-current: a fraction of noninductive current is still present, as it is used as a degree of freedom to ensure that initially, the sum of inductive and non-inductive contributions to the plasma current density are indeed equal to the current density profile provided by the ideal MHD equilibrium. It should also be noted that, by modifying the dynamic of the current profile, we can expect the $\alpha_{C D}$ parameter to have an impact on the dynamics of the sawteeth. This is plotted in the bottom panel of figure 3, where the sawtooth period $\tau_{S}$ is plotted as a function of $\alpha_{C D}$. It appears from this result that increasing the fraction of non-inductive current leads to more frequent sawteeth. We find that for a given set of parameters, the shear on $q=1$ at the time of the sawtooth crash (later denoted as $s_{1, \text { crit }}$ ) remains fairly constant throughout the different sawteeth, as evidenced in the middle panel of figure 3, where for a given value of $\alpha_{C D}$, a low variability of the critical shear is highlighted. The existence of such a critical shear for characterizing the onset of the sawtooth instability is consistent with both theoretical works $[32,33]$ and experimental analyses [57]. We however cannot compare the critical shear obtained in our simulations with the simple analytical expressions derived in [32] or [33]. Indeed, in these analytical approaches, the critical shear is proportional to the density gradient, which is, in our case, fluctuating around zero. This justifies the study of section 5 using a reduced model, without computing one of these analytical expressions of the critical shear. We however find that $s_{1, \text { crit }}$ does depend on the value of $\alpha_{C D}$. A relative good fit of the data is obtained using

$$
s_{1, \text { crit }}=0.19+0.03 \times \alpha_{C D}
$$

This scaling of the critical shear with $\alpha_{C D}$ will be used later in this paper (section 5).

\section{Interaction of sawteeth with external heating or current-drive}

We now focus on the response of sawteeth to external heating or current-drive provided by external sources. We investigate these two effects separately so as to be able to

quantify the impact of each one. It should be noted that in this section, the power $\left(S_{R F}^{s}\right)$ or current $\left(J_{R F}^{s}\right)$ depositions are set with Gaussian profiles in the radial direction, the depositions being constant in the poloidal and toroidal directions (see equations 11 and 12). In particular, the current and power depositions are not computed self-consistently using ray-tracing and Fokker-Planck absorption modeling. Thus, any possible effects that the modification of density or temperature by the sawteeth could have on wave absorption and trajectory in the plasma are not captured. The sawteeth will however still affect the current and power depositions by modifying the magnetic field structure, 


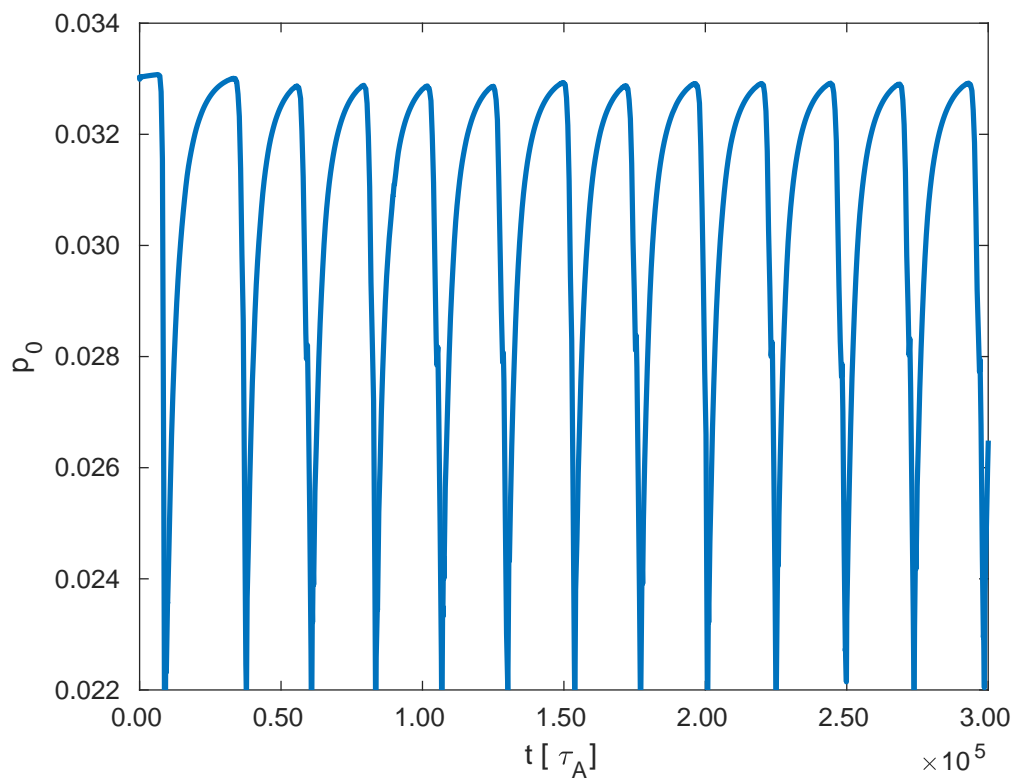

Figure 4. Evolution of the central pressure $p_{0}$ in the reference case, in the absence of RF-current deposition, with $\alpha_{C D}=1$. Results are from XTOR simulations.

thus affecting the propagation of the depositions along the magnetic field lines (see the parallel diffusion terms in equations 5 and 6).

\subsection{Control of sawtooth period by current deposition}

In this first part, we choose $\alpha_{C D}=1$. Simulations lead to a steady sawtooth cycle, as shown in figure 4 , where we have plotted the evolution of the central pressure, denoted $p_{0}$ in the following, along time in the absence of current and power deposition. We observe a set of well resolved, repeatable sawteeth, with a sawtooth period $\tau_{S, 0} \approx 2.33 \times 10^{4} \tau_{A}$. The inversion radius of the sawteeth is $\rho_{i n v} \approx 0.34$. Once the sawtooth cycle is well established, at $t \approx 100000 \tau_{A}$, we switch on the source term $J_{R F}^{s}$ that appears in equation 5. This term is specified as

$$
J_{R F}^{s}(\rho, \theta, \varphi)=J_{R F}^{s, 0} \times \mathrm{e}^{-\frac{\left(\rho-\rho_{R F}(t)\right)^{2}}{2 \sigma_{\rho}^{2}}}
$$

The deposition is continuous in time, with $\sigma_{\rho}=0.015$. In the following, $I_{R F}$ will denote the total RF-driven current, while $I_{P}$ denotes the total plasma current. Positive values of $I_{R F}$ correspond to co-current, indicating that the current is driven in the same direction as the plasma current. Negative values denote counter-current. In figure 5, we have plotted time traces of the central pressure $p_{0}$ for different co-current deposition locations, the total injected current being kept constant.

In figure 6, the profile of the safety factor $q$ is plotted for different locations of the current deposition, at different times during a sawtooth. The reference case without current deposition is also plotted. We observe in particular that after the crash, $q \geq 1$, which indicates that in these simulations, a full magnetic reconnection is observed. This 
Non-linear MHD simulations of sawteeth and their control by current and power depositions10

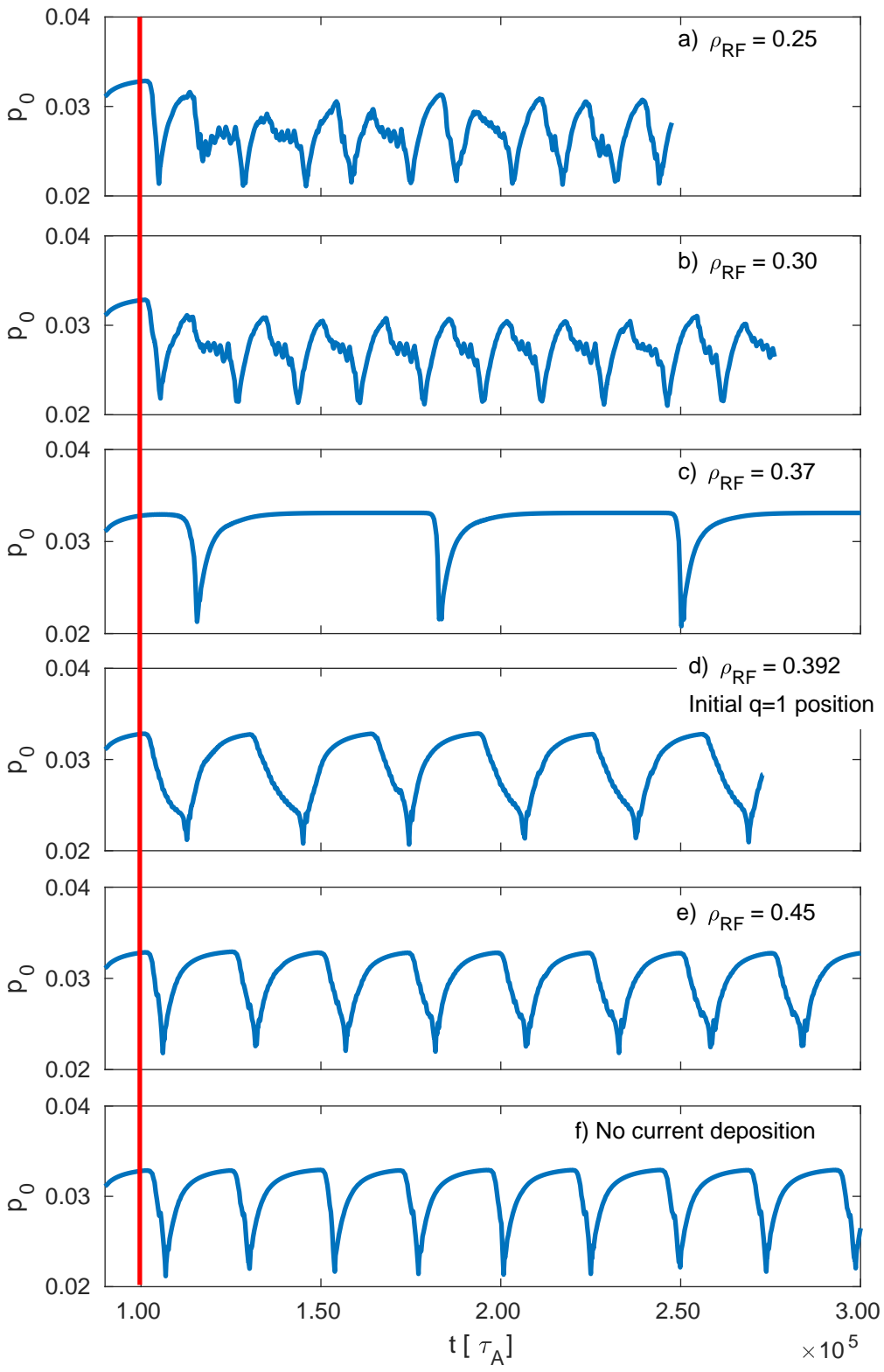

Figure 5. Evolution of the central pressure $p_{0}$. Depending on the radius of the current deposition, the shape and period of the sawteeth are altered. The vertical red bar indicates the moment at which the current deposition is switched on. At equilibrium, $q=1$ is located at $\rho=0.392$. The current deposition is defined so that $I_{R F} / I_{P}=1 \%$. Due to the large period of certain cases $\left(\rho_{R F}=0.37\right.$ for instance), all simulations are not run up to the same time. 
is the case for all the simulations presented in this paper, although we do not show all the $q$-profiles, and more generally for all sawtooth crashes simulated so far with the XTOR-2F code. Experimental observations do report indications of full reconnection $[58,59]$ but the central safety factor seems to remain below unity in other cases $[60,8,61]$. Although the physical model used in XTOR could miss the key element making possible an incomplete reconnection, it has to be said that we have not explored so far a large variety of equilibria. In incomplete reconnection situations, the dynamics of the $q$ profile during the crash is likely different and so should be the MHD activity. These cases are therefore not covered by our simulations.

In figure 7 , we have plotted the modification of the sawtooth period as a function of the current deposition radius, for different values of the $\mathrm{RF}$ current. We observe that for co-current depositions located outside the inversion radius $\rho_{i n v}$, the current deposition leads to an increase of the sawtooth period, while for deposition inside $\rho_{i n v}$ the sawtooth period is decreased. This observation is in good agreement with experimental observations and predictions from simpler models [43]. As one can see, increasing the current amplitude leads to an amplification of the effect. For counter-current depositions $\left(I_{R F} / I_{P}=-1 \%\right)$, we see an opposite behavior: depositions inside the inversion radius tend to increase the sawtooth period, while deposition outside tend to decrease it. Again, this is consistent with experimental observations [37, 39, 24]. Interestingly, we do not observe the strong period peaking observed around the inversion radius in the co-current simulations of same amplitude.

The sawtooth onset is often considered to be governed by the evolution of the shear on $q=1$, the crash occurring when a critical value $s_{1, \text { crit }}$ is reached. In the top panel of figure 8 , we have plotted the time evolution of the shear on $q=1$ for different locations of the RF current depositions. The bottom panel of figure 8 shows the critical shear on $q=1$ at the sawtooth onset for different radial locations of the current deposition for a co-current deposition. We observe that while the current deposition tends to modify the critical shear at which sawteeth are triggered, its main effect appears to be on the dynamics of the shear itself. The evolution of the shear towards its critical value is much slower in the case of depositions near or outside the inversion radius. This is fully compatible with the observation of longer sawtooth periods for these cases. Once the critical value is reached, a crash occurs abruplty. On the contrary, for depositions inside the inversion radius, we observe long-lived oscillations of the shear on $q=1$ during the crash.

\subsection{Control of sawtooth period by power deposition}

We now move on to the stabilization of sawteeth by localized heating. In this part, in order to maximize the contribution of the ohmic part to the total plasma current, we set $\alpha_{C D}=0.0$. This set of parameters leads to a cycle of sawteeth, with a sawtooth period $\tau_{S, 0} \approx 2.77 \times 10^{4} \tau_{A}$. The inversion radius of the sawteeth is again $\rho_{\text {inv }} \approx 0.34$. We add a power density source term, $S_{R F}$, as specified in equation 3 . This term is evolved 
Non-linear MHD simulations of sawteeth and their control by current and power depositions12
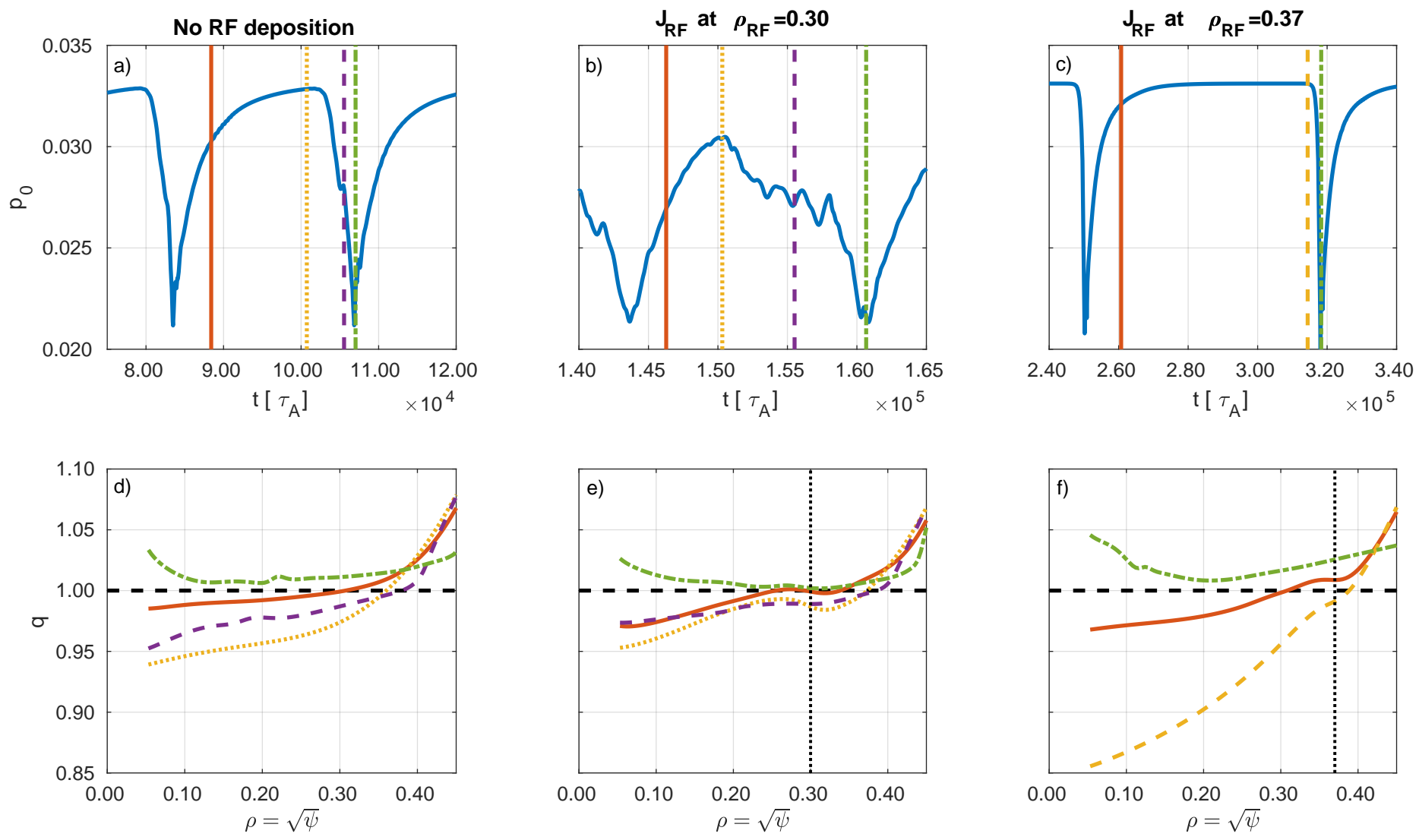

Figure 6. (top line) Evolution of the central pressure as a function time, for $\alpha_{C D}=1$ and in the absence of RF deposition (left, a), in presence of current deposition at $\rho_{R F}=0.30\left(I_{R F} / I_{P}=10 \%\right)$ (middle, b), in presence of current deposition at $\rho_{R F}=0.37\left(I_{R F} / I_{P}=10 \%\right)$ (right, c). (bottom line, d), e) and f)) Profiles of the safety factor $q$ for the different cases plotted on the top line. The different colors and linestyles correspond to different times at which the $q$ profile are plotted. The vertical lines of corresponding colors and styles in the top plots indicate when the $q$-profiles snapshot has been taken with respect to the sawtooth. The red ful line corresponds to a $q$ profile plotted during the ramp-phase of the sawtooth. The yellow-dotted line corresponds to a $q$ profiles plotted when the central pressure $p_{0}$ is near its maximum. The purple dashed line corresponds to a $q$ profile plotted during the sawtooth crash. It is not plotted for the case at $\rho_{R F}=0.37$ since the crash is very rapid. The green dash-dotted line corresponds to $q$ profile after the crash, when $p_{0}$ is minimum. In particular, one notes that in all cases, after the crash, one has $q>1$ everywhere. The horizontal black dashed lines in the bottom plots indicate $q=1$. The vertical black dotted lines in the bottom plots indicate the position of the current deposition. Due to the difficulty to compute the $q$ profile in the center when the magnetic axis is not exactly on the center of the XTOR computation grid, values of $q$ inside $\rho=0.05$ are not plotted. 


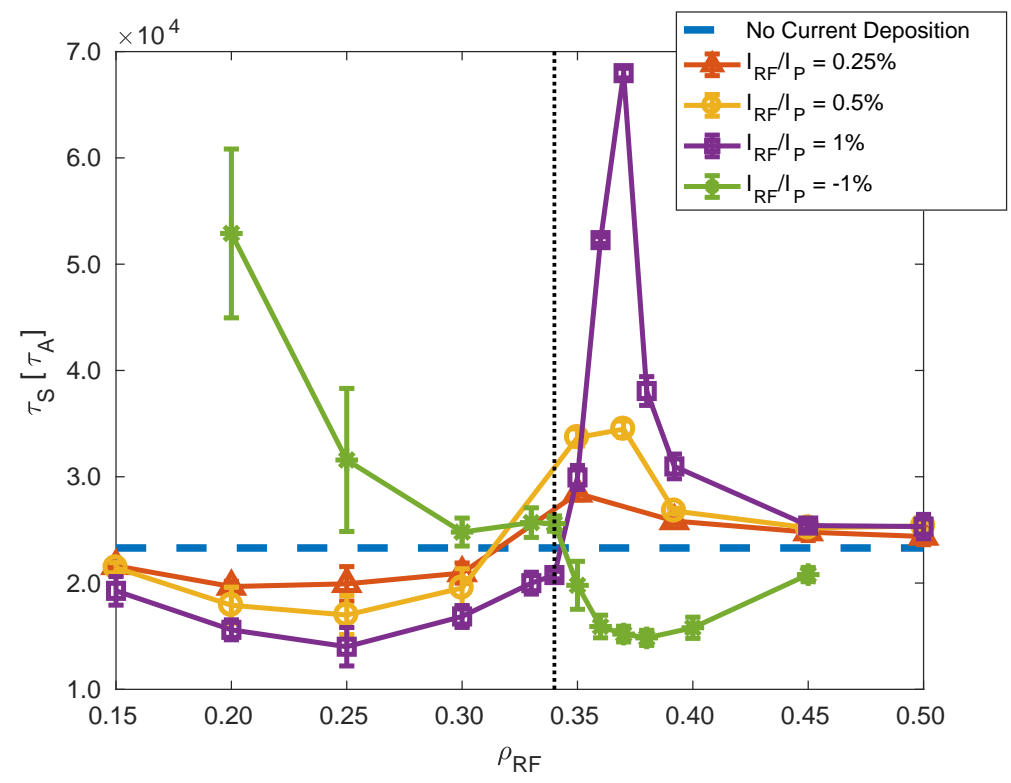

Figure 7. Evolution of the sawtooth period depending on the radial location of the ECCD current deposition, for different values of the current intensity $\left(\alpha_{C D}=1\right)$. The horizontal dashed line indicates the sawtooth period $\tau_{S, 0}$ in the absence of current deposition. The vertical dotted line indicates the position of the inversion radius $\rho_{i n v}$.
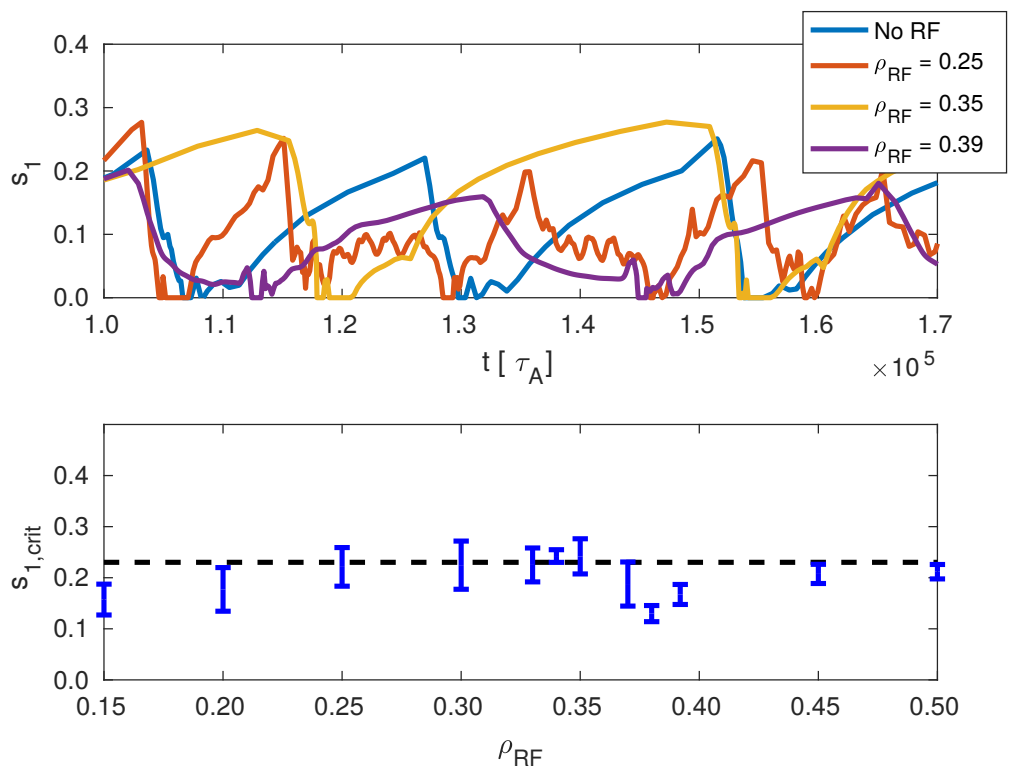

Figure 8. (top) Time traces of the shear $s_{1}$ on $q=1$ for different positions of the current deposition, for a given RF current (1\% of the plasma current), and for a case without current injection. (bottom) Critical shear $s_{1, \text { crit }}$ on $q=1$ at the triggering of the sawtooth for different radial location of the current deposition. The critical shear in the case without current deposition is indicated by the dashed-line, with a critical shear $s_{1, \text { crit }} \approx 0.22$. In this figure, $I_{R F} / I_{P}=1 \%$. 
Non-linear MHD simulations of sawteeth and their control by current and power depositions14

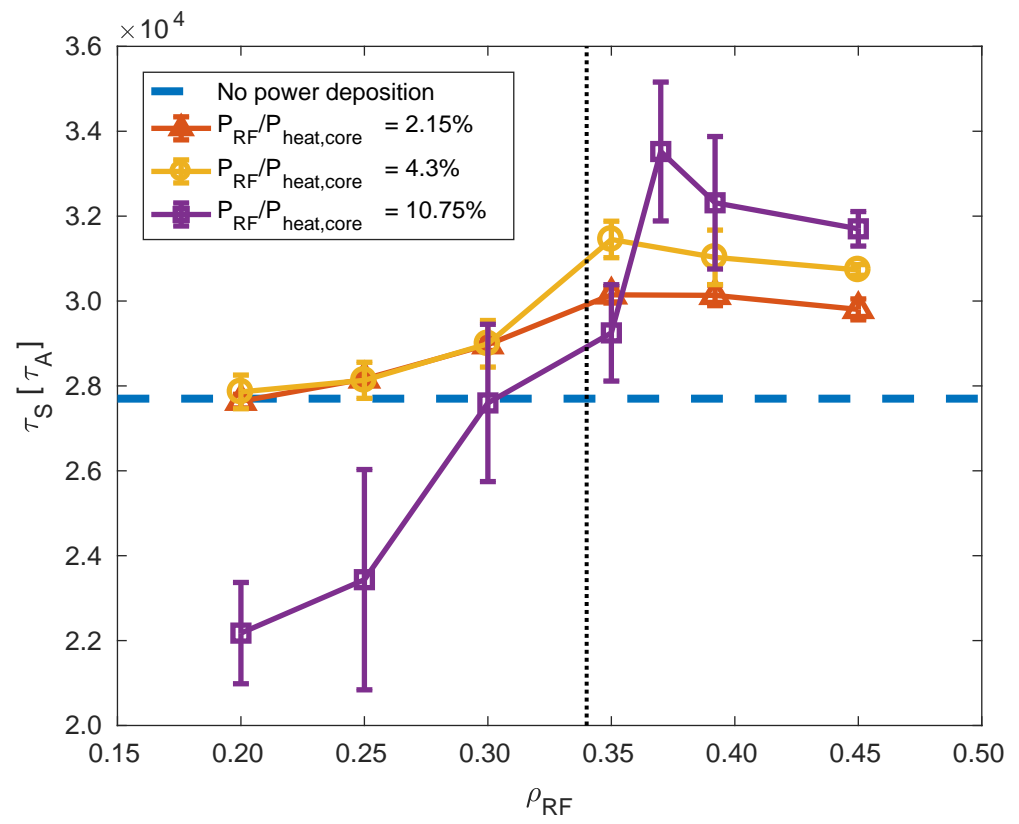

Figure 9. Evolution of the sawtooth period depending on the radial location of the power deposition, for different values of the power source intensity. In those cases, $\alpha_{C D}=0.0$. The horizontal dashed line indicates the sawtooth period $\tau_{S, 0}$ in the absence of current deposition. The vertical dotted line indicates the position of the inversion radius $\rho_{i n v}$.

following equation 6 , where $S_{R F}^{s}$, which mimics the power deposition that would result from the external heating source used to act on the sawteeth, is specified as

$$
S_{R F}^{s}(\rho, \theta, \varphi)=S_{R F}^{s, 0} \times \mathrm{e}^{-\frac{\left(\rho-\rho_{R F}(t)\right)^{2}}{2 \sigma_{\rho}^{2}}}
$$

The deposition is continuous, and $\sigma_{\rho}=0.015$. In the following, the quantity of power deposited in the plasma will be indicated by the ratio $P_{R F} / P_{\text {heat,core }}$ where $P_{R F}$ is the volume integral of $S_{R F}^{s}(\rho, \theta, \varphi)$ and represents the total power deposited by our external heating source used to act on the sawteeth, while $P_{\text {heat,core }}$ is the volume integral of the $S_{H}$ term in equation 3 for $\rho<0.5$, and represents the power used to "heat" the core plasma and maintain the prescribed equilibrium pressure profile. The results are more complex than in the co-current case. Similarly to co-current-drive case, we observe that depositions outside the inversion radius $\rho_{i n v}$ lead to longer sawteeth than depositions inside $\rho_{i n v}$. However, if the deposited power is too low, then all depositions lead to a longer sawtooth period than in the absence of power deposition. This is plotted in figure 9, which shows the modification of the sawtooth period as a function of the power deposition radial location, for different values of the $\mathrm{RF}$ power. It should be noted that the effect in power seems less pronounced than in the case of current deposition only because the level of power injected is relatively low, and so the current profile is less affected than in the case of direct current deposition. In addition to that, the power deposition is not only enlarged by the effect of $\chi_{\perp}^{R F}$, but the pressure perturbation it produces is further broadened by the perpendicular diffusivity $\chi_{\perp}$ in equation 3 . 
Non-linear MHD simulations of sawteeth and their control by current and power depositions15

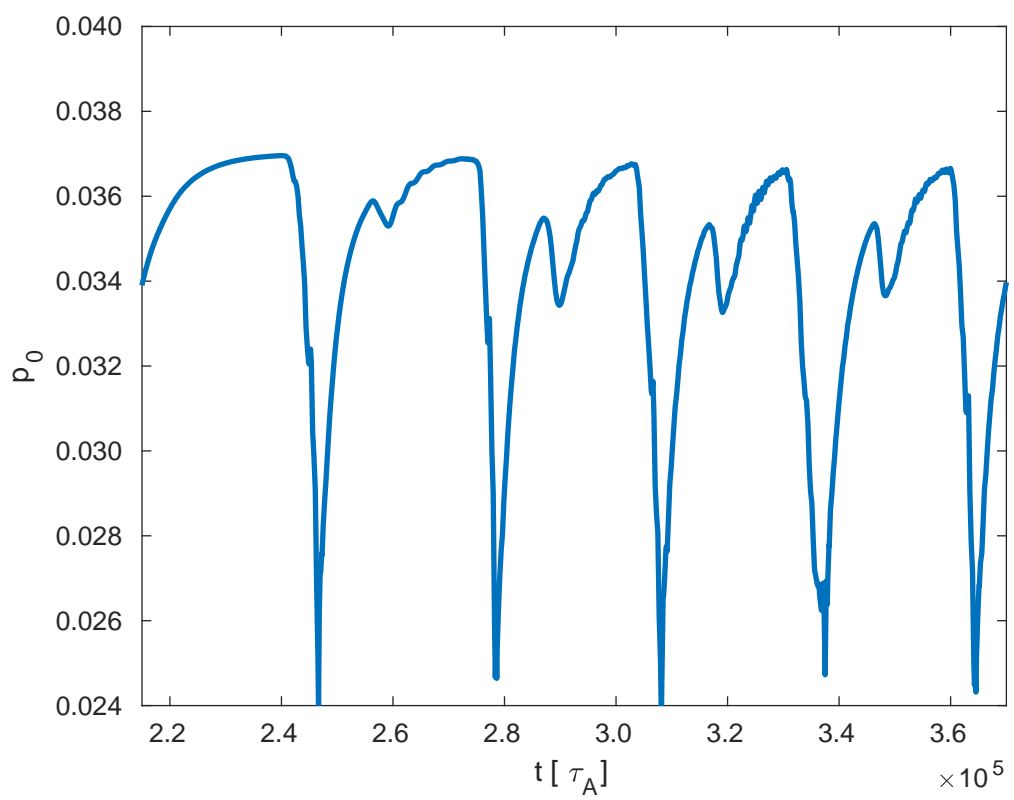

Figure 10. Evolution of the central pressure $p_{0}$ in localized heating case, $\alpha_{C D}=0$. The power deposition is centered on $\rho_{R F}=0.35$, and $P_{R F} / P_{\text {heat,core }} \approx 10 \%$. Notice the partial crashes occurring during the ramp phases of the sawteeth.

\subsection{Modification of sawtooth shape by current or power deposition}

As it can be seen in figures 5 and 10, the localized current drive or heating leads to a modification of the shape of the sawteeth. This effect is also observed experimentally, where a large variety of sawtooth shapes has been observed [62, 35]. This modification of the shape is important, as it could play a role on the transport of tungsten during the collapse [13]. In this part, we give preliminary observations. We focus on the case with full current-drive $\left(\alpha_{C D}=1\right)$. We observe that in several cases, corresponding to injection at $\rho_{R F}=0.20,0.25$, and 0.30 , instead of an abrupt crash, we have either a longer crash marked by oscillations or even a two-step crash, during which a first partial crash of the core pressure is followed by a period of oscillation of the central pressure, until a second crash occurs, after what the pressure is flattened in the core region and a new cycle begins. If one plots the radial position of the maximum of the temperature (labeled as "displacement" and denoted $\max _{\rho} T$ ), which is a good indicator to follow the position of the hot-core during its expulsion, as done in figures 11 and 12, it appears clearly that the expulsion of the core "pauses", and that the hot core keeps rotating while staying inside the inversion radius until at some point, this rotation ends and the hot core is finally expelled. This behavior has been observed on Asdex-Upgrade for example[9].

The precise understanding of this modification of the sawtooth shape by the ECCD remains however unsolved. However, one may think that a likely player is a modification of the linear stability of the $(1,1)$ mode due to modification of the current and pressure profiles by the action of current and power depositions. 
Non-linear MHD simulations of sawteeth and their control by current and power depositions16
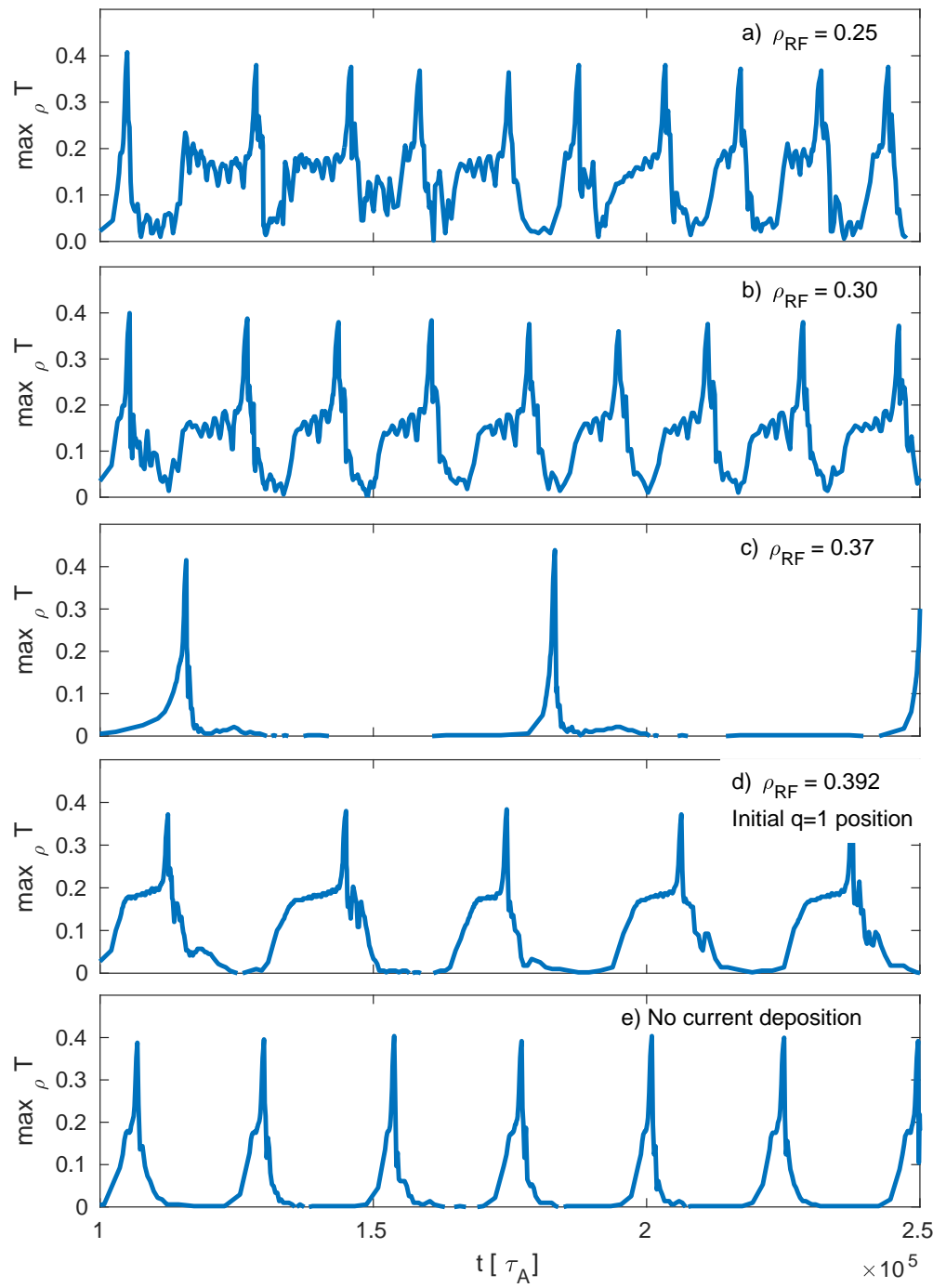

Figure 11. Evolution of the radial location of the maximum of the temperature $\max _{\rho} T$ as a function of time, for different locations of the current deposition $\left(\alpha_{C D}=1\right.$, $\left.I_{R F} / I_{P}=1 \%\right)$. One observes that depending on the location of the deposition, the radial trajectory of the hot core differs. In particular, for certain depositions, it features a long oscillatory period around $\rho=0.2$, that is, midway from its ejection value at $\rho \approx 0.4$.

\subsection{Role of the current source terms on the dynamics of sawteeth}

By changing the current source term in the expression of the Ohm's law, one would expect the dynamics of the sawteeth to be impacted. In this section, we describe the effect of changing $\alpha_{C D}$ on the dynamics of the sawteeth, with and without additional current-drive. We observe that increasing the value of $\alpha_{C D}$, that is, increasing the part of non-ohmic current, leads to a slight reduction of the sawtooth period (as shown in figure 3) but their amplitudes remain similar. We now investigate the effect of $\alpha_{C D}$ on the possibility to control sawteeth with current or power depositions. Since the sawtooth period varies as we change $\alpha_{C D}$, we define the relative modification of the 

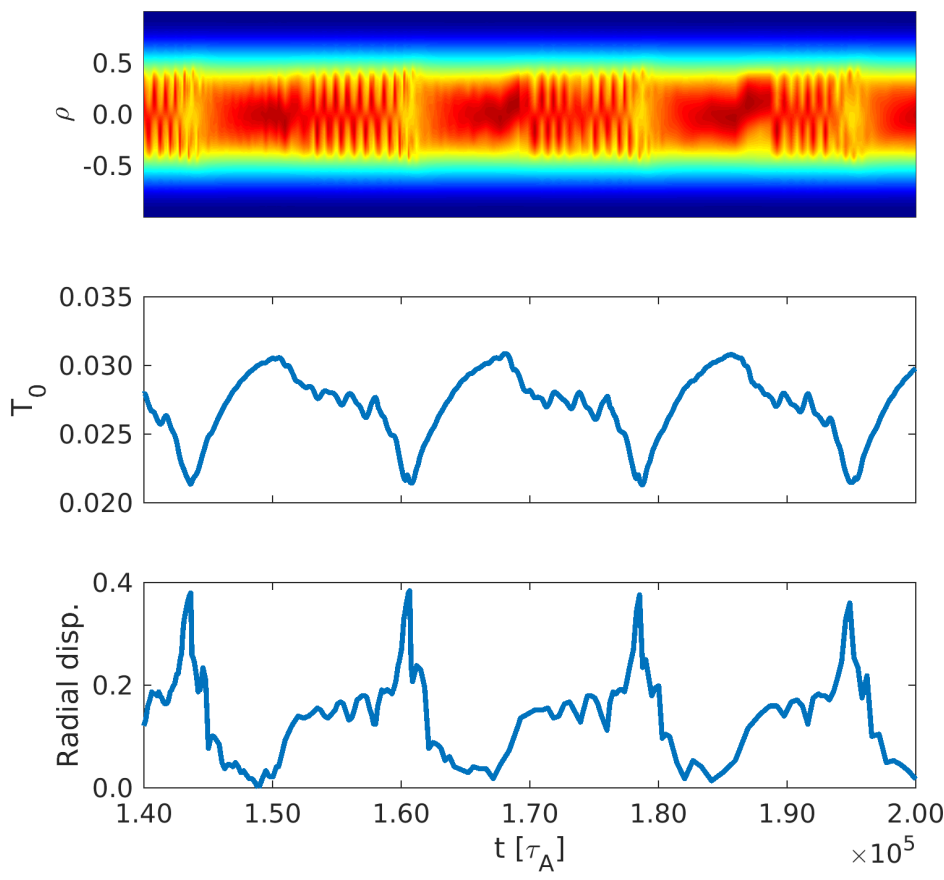

Figure 12. (top) Evolution of the temperature mid-plane profile during sawtooth activity in presence of a current deposition at $\rho_{R F}=0.30$, such that $I_{R F} / I_{P}=1 \%$. The long-lived oscillations of the hot-core can be seen during the crash phase. Precursor oscillations are also visible before the crash. (middle) Evolution of the central temperature $T_{0}$. Again, the oscillations of the hot-core are visible on this time trace. (bottom) Evolution of the radial position of the temperature maximum (in a poloidal cross-section). The long-lived oscillations observed on the temperature profile coincide with the hot-core remaining near $\rho \approx 0.2$, before being expelled during the final phase of the crash.

sawtooth period $\Delta \tau_{S}$ of the sawtooth period, defined as

$$
\Delta \tau_{S}=\frac{\tau_{S}-\tau_{S, 0}}{\tau_{S, 0}}
$$

where $\tau_{S, 0}$ is the sawtooth period in the absence of current deposition $\left(J_{R F}=0\right)$, and is a function of $\alpha_{C D}$. In figure 13, $\Delta \tau_{S}$ is plotted as a function of the deposition radial location for a given value of the current $\left(I_{R F} / I_{P}=1 \%\right)$, for different values of $\alpha_{C D}$. It appears that while there are some variations, the behavior remains overall similar. This is consistent with the fact that the deposited current directly acts on the current profile and is thus able to impact the magnetic shear whatever the value of $\alpha_{C D}$ is. This picture is however different in the case of power depositions, as shown in figure 14. In this situation, it appears that only for $\alpha_{C D}=0$ the period can effectively be reduced. For $\alpha_{C D} \neq 0$, it appears that the period is increased, probably because of the overall modification of the pressure profile by the power deposition. Let us first comment the case $\alpha_{C D}=1$. This corresponds to a purely non-inductive plasma current and therefore, the modification of the temperature profile by the power deposition does 
Non-linear MHD simulations of sawteeth and their control by current and power depositions18

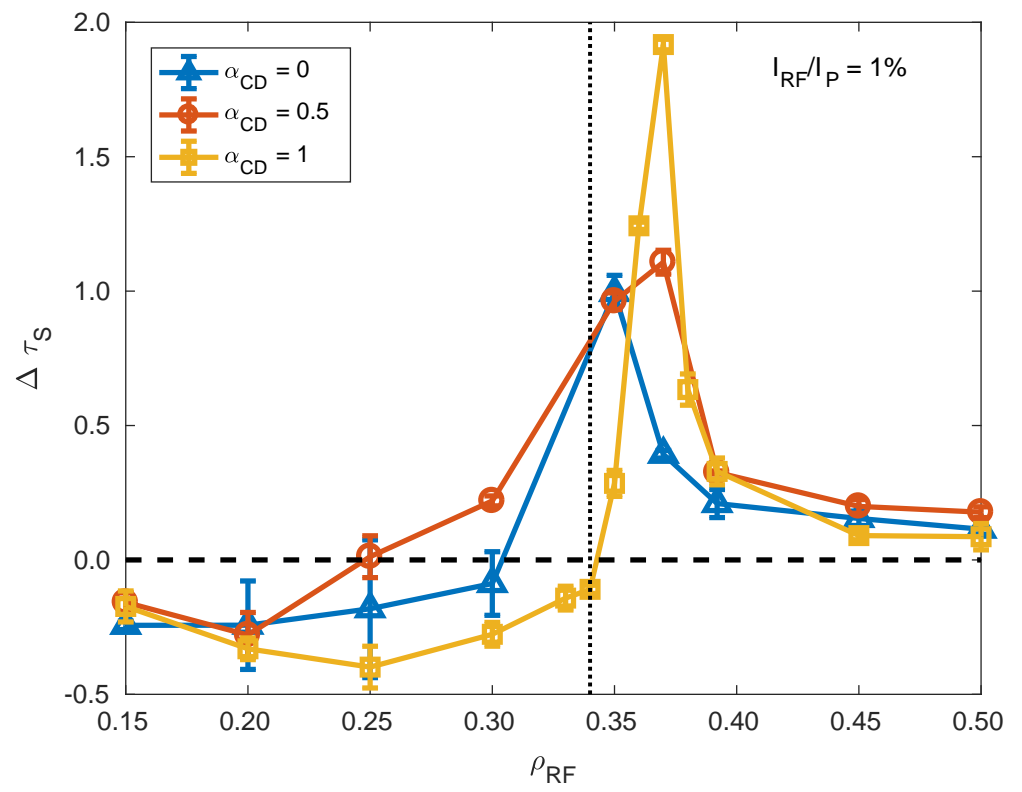

Figure 13. Relative modification of the sawtooth period $\Delta \tau_{S}$ for current injection at different values of the radial location $\rho_{R F}$ and for different values of $\alpha_{C D}$. The injected current is kept constant (co-current, $I_{R F} / I_{P}=1 \%$ ). As plotted in the figure, the behavior remains similar for the different values of $\alpha_{C D}$. The horizontal dashed line indicates $\Delta \tau_{S}=0$. The vertical dotted line indicates the position of the inversion radius $\rho_{\text {inv }}$.

not directly affect the current profile, and thus the shear, since the plasma current remains hooked to its equilibrium value $\mathbf{J}_{C D}$. However, the modification of $\eta$ with the temperature will affect the relaxation of the current profile evolution towards its equilibrium value $\mathbf{J}_{C D}$. As the temperature is increased, and thus $\eta$ is decreased, the resistive timescale is increased, and thus the relaxation of the current profile will be slower. This is consistent with what is observed in figure 14. Another manifestation of the pressure profile modification by the power injection is through the modification of the diamagnetic effects, which are known to be stabilizing in the linear phase of the sawtooth, thus also providing an explanation to the overall increase of the sawtooth period. In the intermediate case $\alpha_{C D}=0.5$, the observed behavior appears to be midway between the $\alpha_{C D}=0$ and $\alpha_{C D}=1$ ones: While the period is mainly increased when the power is deposited near the inversion radius (as seen with $\alpha_{C D}=0$ ), the period is always increased with respect to the absence of power deposition, as seen with $\alpha_{C D}=1$. It should be noted that in our computations, the bootstrap current is not included. Since this current scales as $\nabla p$, if included in the simulation, the current profile would be affected by the power deposition through the modification of the bootstrap contribution.

\section{Modeling of the sawtooth period evolution with a critical shear model}

In the bottom panel of figure 8 , we have plotted the shear $s_{1, \text { crit }}$ at which sawteeth 
Non-linear MHD simulations of sawteeth and their control by current and power depositions19

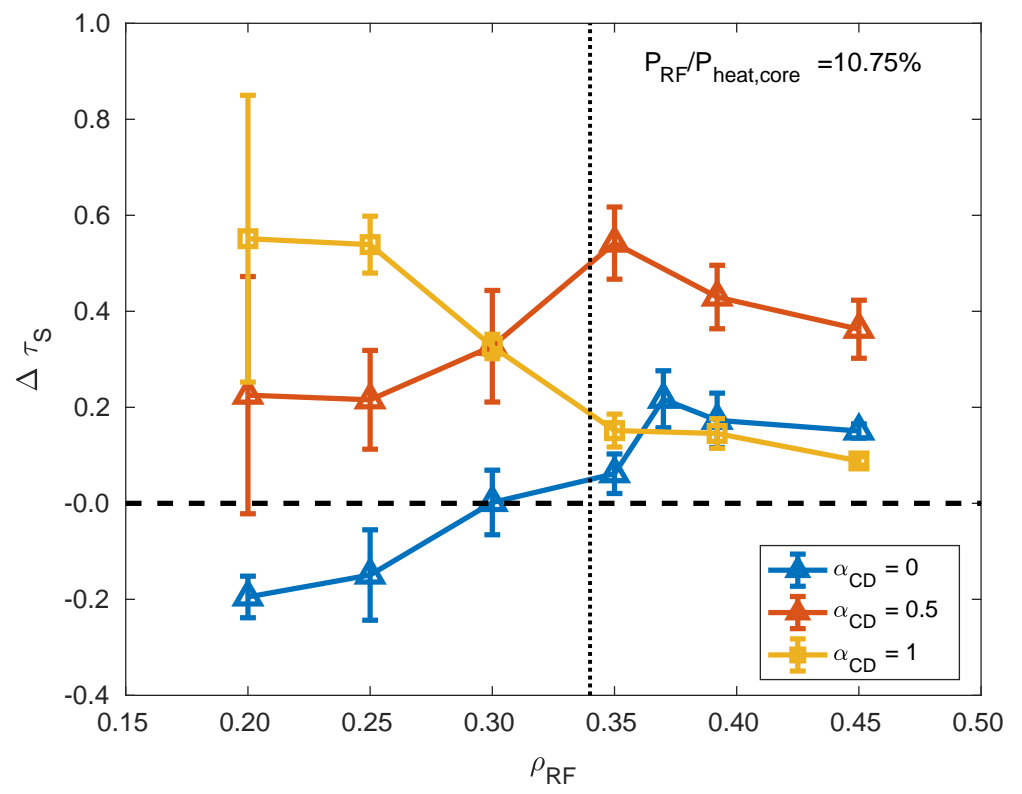

Figure 14. Evolution of the sawtooth period for power injection at different values of the radial location $\rho_{R F}$, the injected power being kept constant $\left(S_{R F} / P_{\text {heat }, \text { core }} \approx\right.$ $10 \%$ ), and for different values of $\alpha_{C D}$. A strong difference can be seen on the period behavior as $\alpha_{C D}$ is increased, which can be explained by the fact that for $\alpha_{C D} \rightarrow 1$, the power injection tends to have less direct effect on the shear and is only affecting the temperature profile, and the dynamics of the current profile, by modifying the local resistive time. The horizontal dashed line indicates $\Delta \tau_{S}=0$. The vertical dotted line indicates the position of the inversion radius $\rho_{i n v}$.

are triggered in different situations (with and without current deposition). The low amplitude of the error bars indicates that the sawtooth crashes occur for a relatively stable value of $s_{1, \text { crit }}$. Thus, the effect of power or current depositions on the sawtooth frequency seems to be consistent with the classical picture of a critical shear-model for the sawteeth $[32,33]$, where the sawteeth are triggered when a critical value of the shear is reached. However, figure 5 reveals that the dynamics of the crash itself is quite complex, with possible long oscillations of the core position (see figures 11 and 12). This behavior is not taken into account in the analytical modeling, and illustrates the interest of 3D, full MHD simulations where the sawtooth dynamics can be described in a self-consistent way. Still, in this section, we introduce a simple 1D model and compare it to our XTOR results in order to check how well they agree despite missing parts of the dynamics. As mentioned earlier, the available expressions derived in the literature for the critical shear are proportional to the density gradient and cannot be applied in our case where the density profile is extremely flat, and its gradient crosses zero during the sawtooth ramp. Thus, for simplicity, we assume the existence of a critical shear based on the empirical observations of figure 8 , and specify it as a constant derived from figure 8. To model the time evolution between the sawtooth crashes, we use simple diffusion equations for the pressure and magnetic field profiles. The crash is triggered when the shear at the location of $q=1$ reaches the critical value $s_{1, \text { crit }}$. Post-crash profiles are 
then computed using the Kadomtsev reconnection model [4]. To do so, we follow an approach similar to the one described in [63]. The evolution of the poloidal magnetic field $B_{\theta}$ is described by

$$
\frac{\partial B_{\theta}}{\partial t}=\frac{\partial}{\partial \rho}\left(\frac{\eta}{\mu_{0} \rho}\left(B_{\theta}+\rho \frac{\partial B_{\theta}}{\partial \rho}\right)-\eta\left(J_{C D}+J_{R F}\right)\right)
$$

where $\rho$ is the normalized radial coordinate. $J_{C D}$ is a current source, the role of which is to restore the equilibrium $J_{\phi}^{e q}$ and $J_{R F}$ corresponds to the current driven by the ECCD. Similarly to what has been presented in section 3 , we define $J_{C D}$ as follows,

$$
J_{C D}=J_{\phi}^{e q}-\frac{\left(1-\alpha_{C D}\right) \eta_{0}^{e q} J_{\phi, 0}^{e q}}{\eta^{e q}}
$$

where $J_{\phi, 0}^{e q}$ is the equilibrium current at $\rho=0$ and $\eta_{0}^{e q}$ is the equilibrium resistivity at $\rho=0$. Like in section $3, \alpha_{C D}$ quantifies the level of non-inductive current. We recall that $\alpha_{C D}=0$ does not imply that all the current is inductively-driven, but that all the current on the magnetic axis $(\rho=0)$ is inductively-driven. In the following, we note $\tilde{E}=\left(1-\alpha_{C D}\right) \eta_{0}^{e q} J_{\phi, 0}^{e q}$. Alongside the evolution of $B_{\theta}$, we evolve the temperature profile assuming a diffusive model

$$
\frac{\partial T}{\partial t}=\chi \nabla^{2}\left(T-T_{i n i}\right)+S_{R F}
$$

where $T_{i n i}$ is the initial temperature profile. Equations 14 and 16 are solved assuming a certain profile of $J_{\phi}^{e q}$, given by the initial $q$ profile, and the initial temperature profile $T_{i n i}$. The boundary conditions used for equation 14 are $B_{\theta}(\rho=1)=B_{\theta, 1}=$ cst et $B_{\theta}(\rho=0)=B_{\theta, 0}=0$, while for equation 16 , we use $T(\rho=1)=T_{1}$ where $T_{1}$ is a constant, and $T^{\prime}(\rho=0)=T_{0}^{\prime}=0$. The resistivity $\eta$ in equation 14 is defined as

$$
\eta=\eta_{0}\left(\frac{T_{0}}{T}\right)^{\frac{3}{2}}
$$

where $T_{0}$ and $\eta_{0}$ are respectively the initial temperature and resistivity at $\rho=0$. In steady-state (neglecting the existence of sawteeth), the temperature (and thus the resistivity) will tend to the profile set by the boundary conditions on $T$. The boundary conditions will also impose the profile of the magnetic field. However, what is of interest for us is the current profile $J_{\phi}$. In the following, we show that the behavior obtained with the 1D model matches the one we imposed in XTOR in section 3. We start by considering the steady-state of equation 14

$$
\frac{\partial}{\partial \rho}\left(\frac{\eta}{\mu_{0} \rho}\left(B_{\theta}+\rho \frac{\partial B_{\theta}}{\partial \rho}\right)-\eta\left(J_{C D}+J_{R F}\right)\right)=0
$$

and thus, using that $J_{\phi}=\frac{1}{\mu_{0} \rho}\left(B_{\theta}+\rho \frac{\partial B_{\theta}}{\partial \rho}\right)$,

$$
\eta\left(J_{\phi}-\left(J_{C D}+J_{R F}\right)\right)=E
$$

where $E$ is a constant. Since the steady state value of $B_{\theta}$ is fully determined by the boundary conditions we impose, $J_{\phi, 1}^{S T}$, the value of $J_{\phi}^{S T}$ at the boundary, is also fixed. Here, $J_{\phi}^{S T}$ denotes the value of $J_{\phi}$ when a steady-state is reached. The value $\eta_{1}^{S T}$ of $\eta^{S T}$ 
at the edge is also set by the boundary condition $T(\rho=1)=T_{1}$. Again, $\eta^{S T}$ denotes the value of $\eta$ when a steady-state is reached. Because $T_{1}$ is a constant, we have $\eta_{1}^{S T}=\eta_{1}^{e q}$ where $\eta_{1}^{e q}$ is the value of $\eta^{e q}$ at the edge. Thus, we have

$$
E=\eta_{1}^{S T}\left[J_{\phi, 1}^{S T}-\left(J_{C D}(\rho=1)+J_{R F}(\rho=1)\right)\right]
$$

which becomes

$$
E=\eta_{1}^{S T}\left[J_{\phi, 1}^{S T}-\left(J_{\phi, 1}^{e q}-\frac{\left(1-\alpha_{C D}\right) \eta_{0}^{e q} J_{\phi, 0}^{e q}}{\eta_{1}^{e q}}\right)\right]
$$

where we have assumed $J_{R F}(\rho=1)=0$ for simplicity (in our simulations, the currentdrive is generally driven well inside $\rho=1$ so that this assumption is valid in the following). Since $J_{\phi, 1}^{S T}=J_{\phi, 1}^{e q}$ (because the boundary conditions imposed on $B_{\theta}$ do not change), we have

$$
E=\left(1-\alpha_{C D}\right) \eta_{0}^{e q} J_{\phi, 0}^{e q}=\tilde{E}
$$

and $E$ is then the "rescaled" electric field. Thus, we have

$$
J_{\phi}-J_{\phi}^{e q} \rightarrow \tilde{E}\left(\frac{1}{\eta^{S T}}-\frac{1}{\eta^{e q}}\right)+J_{R F}
$$

In a full non-inductive current case $\left(\alpha_{C D}=1\right), \tilde{E}=0$ and therefore

$$
J_{\phi}^{S T}=J_{\phi}^{e q}+J_{R F}
$$

Thus, in the full non-inductive current case, the current density is hooked to the equilibrium value $J_{\phi}^{e q}$ set by $J_{C D}$, like in XTOR. When $\alpha_{C D} \neq 0$, then $\tilde{E} \neq 0$. In that case, if additional heating is provided to the plasma, so that $\eta^{S T}<\eta^{e q}$, then one has

$$
J_{\phi}^{S T}=J_{\phi}^{e q}+\tilde{E}\left(\frac{1}{\eta^{S T}}-\frac{1}{\eta^{e q}}\right)+J_{R F}>J_{\phi}^{e q}+J_{R F}
$$

which, again, is the expected behavior in the presence of a fraction of ohmic current. Therefore, in steady-state, this model yields the same behavior as the model that has been developed for XTOR in section 3. Because of the nonlinear nature of the problem, it is more straightforward to solve the system of equations 14 and 16 with an explicit time scheme. In order to enhance the numerical stability and take larger time steps, a stabilized first order Runge Kutta method is used [64]. Using the Kadomstev model, the profiles of the poloidal magnetic field and temperature post-crash are computed and used as the initial condition $(t=0)$. This means that the equilibrium is assumed to be unstable to the sawtooth. We then let the poloidal magnetic field and temperature profiles evolve from their post-crash value to their equilibrium value and we follow the evolution of the shear on the $q=1$ surface. When the critical shear on $q=1$ is reached then a crash is triggered, and the temperature and poloidal magnetic field profiles are modified using the Kadomstev model to mimic the effect of a sawtooth. The cycle then restarts, and after a few sawtooth crashes, we consider the sawtooth cycle as established and compute the sawtooth period. In figure 15, we have plotted the evolution of the sawtooth period as a function of $\alpha_{C D}$, as returned by the model in the absence of source 
Non-linear MHD simulations of sawteeth and their control by current and power depositions22

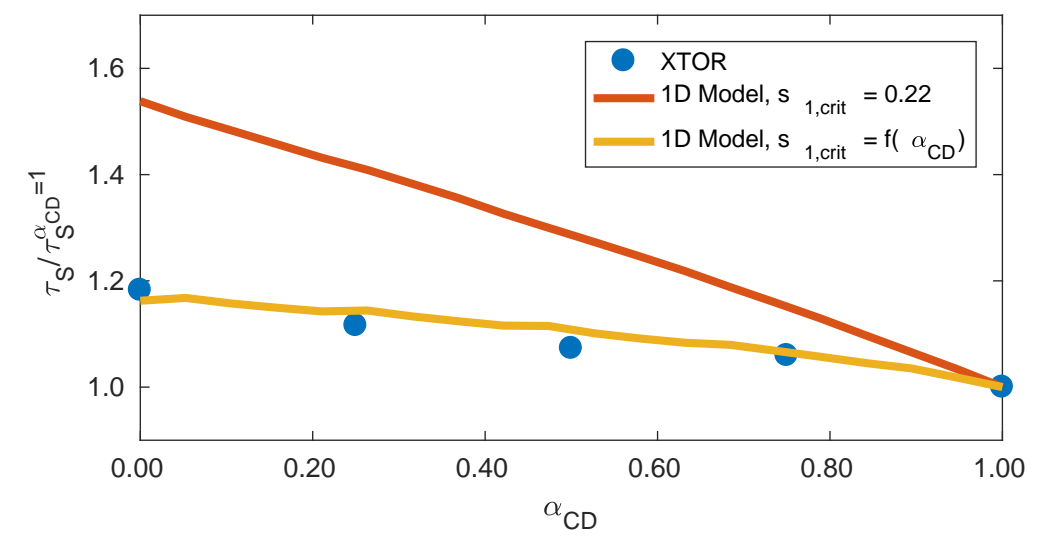

Figure 15. Evolution of the sawtooth period given by XTOR and by the model developed in equations $14-16$, as a function of $\alpha_{C D}$. The period $\tau_{S}$ is normalized to its value for $\alpha_{C D}=1$. Two different models are used for the critical shear: a fixed values $\left(s_{1, \text { crit }}=0.22\right)$ or a value depending on $\alpha_{C D}$ based on the scaling observed in XTOR and given by equation 10 .

terms $\left(J_{R F}=S_{R F}=0\right)$. We observe that the period decreases as $\alpha_{C D}$ is increased, which is reminiscent of the observations made in XTOR and plotted in figure 3 . When taking a shear depending on $\alpha_{C D}$, using the fit given by equation 10, a good agreement is found between the model and XTOR.

We now include current and power depositions in the model. The source terms are defined as

$$
J_{R F}=J_{R F}^{0} \exp \left(-\frac{\left(\rho-\rho_{R F}\right)^{2}}{2 \sigma_{\rho}^{2}}\right)
$$

and

$$
S_{R F}=S_{R F}^{0} \exp \left(-\frac{\left(\rho-\rho_{R F}\right)^{2}}{2 \sigma_{\rho}^{2}}\right)
$$

By varying $\rho_{R F}, J_{R F}^{0}, S_{R F}^{0}$ or $\sigma_{\rho}$, it is then possible to infer the influence of the current or power deposition location, amplitude or width on the sawtooth period. In the following, we compare the results given by this model with the results obtained with XTOR in two cases:

(i) The full current-drive case $\left(\alpha_{C D}=1\right)$. In that case, we only test the influence of the current drive, and $S_{R F}=0 . \quad J_{\phi}$ is hooked to $J_{C D}+J_{R F}$, where $J_{C D}$ is the equilibrium profile of $J_{\phi}$. Therefore, the modification of $\eta$ with the temperature only impacts the dynamics of the current profile evolution, not the steady-state value to be reached.

(ii) The $\alpha_{C D}=0$ case. In that case, we only investigate the effect of the power source, and $J_{R F}=0$. This is the case that corresponds to what one would expect for inductive current. The change in the resistivity caused by the evolution of the temperature profile will be reflected on the steady-state value of $J_{\phi}$. 
It should be noted that in XTOR, the current and power source terms are defined in a way similar to equations 26 and 27, as shown in equation 11. However, in XTOR, the quantity that is conserved for the RF-driven current is $I_{R F}=\int_{S} J_{R F}^{s} \mathrm{~d} S$, which

represents the total RF-driven current. $J_{R F}^{s, 0}$ (see equation 11) is thus adjusted to conserve $I_{R F}$ through the different source locations and widths. In the following scan with the model presented in equations 14-16, we apply the same idea and conserve the quantities $\int \rho J_{R F}(\rho) \mathrm{d} \rho$ and $\int \rho S_{R F}(\rho) \mathrm{d} \rho$. The initial temperature and current profiles used in XTOR are used in the model. As for the source terms, in XTOR the width of the source is set to $\sigma_{\rho}=0.015$, but the source term undergoes a radial broadening due to the presence of a non-vanishing $\chi_{\perp}^{R F}$. To account for this, we perform two simulations with the reduced model, one using $\sigma_{\rho}=0.015$, and the other one using a higher broadness $\sigma_{\rho}=0.030$, which would correspond to the highest broadening observed in the simulations. The critical shear is taken as $s_{1, \text { crit }}=0.22$, similar to what is observed in XTOR simulations (figure 8). The result of the comparison of the model with the XTOR results is displayed in figure 16, where we plot $\Delta \tau_{S}$ as a function of $\rho_{R F}$. It appears that the results are in good qualitative agreement. We also observe a reasonable quantitative agreement, the XTOR simulations lying between the case with $\sigma_{\rho}=0.015$ and the case $\sigma_{\rho}=0.030$. The fact that the model is able to reproduce the tendencies observed in XTOR is an indicator that a critical shear model is well-adapted to describe the physics at play in our simulations, despite the fact that the sawtooth shape is strongly MHD-dependent for $\rho_{R F}<\rho_{q=1}$ in the simulations. While the 1D model assumes a very simplified form for the reconnected $q$-profile shape after the crash, in the simulations, we observe a complex $q$ profile (and thus magnetic shear profile) which remains non-monotonous during the sawtooth recovery. While it is interesting to notice that the $1 \mathrm{D}$ model with Kadomtsev reconnection is still able to provide a fairly correct estimate of the period despite lacking a correct description of the reconnection process observed in XTOR, this emphasizes the need of full MHD simulations. In particular when we compare the results of the model in the heating cases. The results are shown in figure 17. Contrary to the current deposition cases, we observe a quantitative disagreement between the model and XTOR, although the general tendency remains more or less similar. This is a sign that the basic critical shear model captures part of the physics at play but misses some important contributions. Interestingly, the results of the model are similar to the XTOR ones plotted in figure 9 when for lower values of $P_{R F}$. This could indicate that several mechanisms are at play, one of them appearing at higher level of power than the other ones.

\section{Destabilization of tearing modes by sawtooth crash}

In this section, we focus on the onset of metastable tearing modes, that is, modes that are linearly stable, but that can be triggered if a perturbation, having the mode helicity, reaches a critical width. As soon as this critical width is reached, the island will grow up to its critical saturation width. It is well-known that sawtooth crashes can be responsible 
Non-linear MHD simulations of sawteeth and their control by current and power depositions24

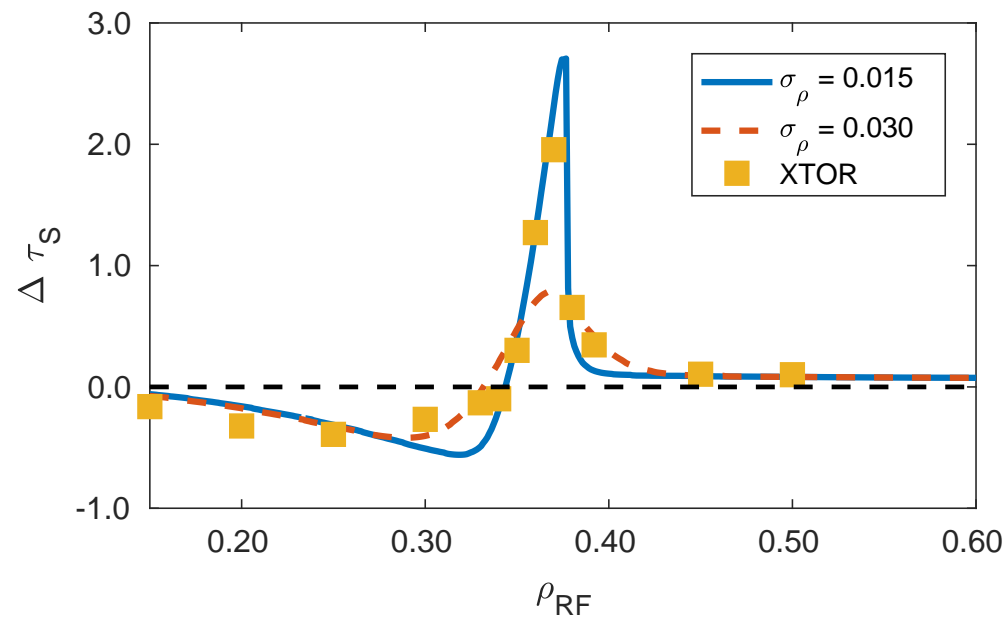

Figure 16. Comparison of the sawtooth period computed by XTOR and the model developed in equations 14-16 for current deposition at different values of the radial location $\rho_{R F}$, the injected current being kept constant $\left(I_{R F} / I_{P}=1.0 \%\right.$ in the XTOR simulations, $\left.\alpha_{C D}=1\right)$. The critical shear has been set to $s_{1, \text { crit }}=0.22$. The power source $S_{R F}$ is set to 0 .

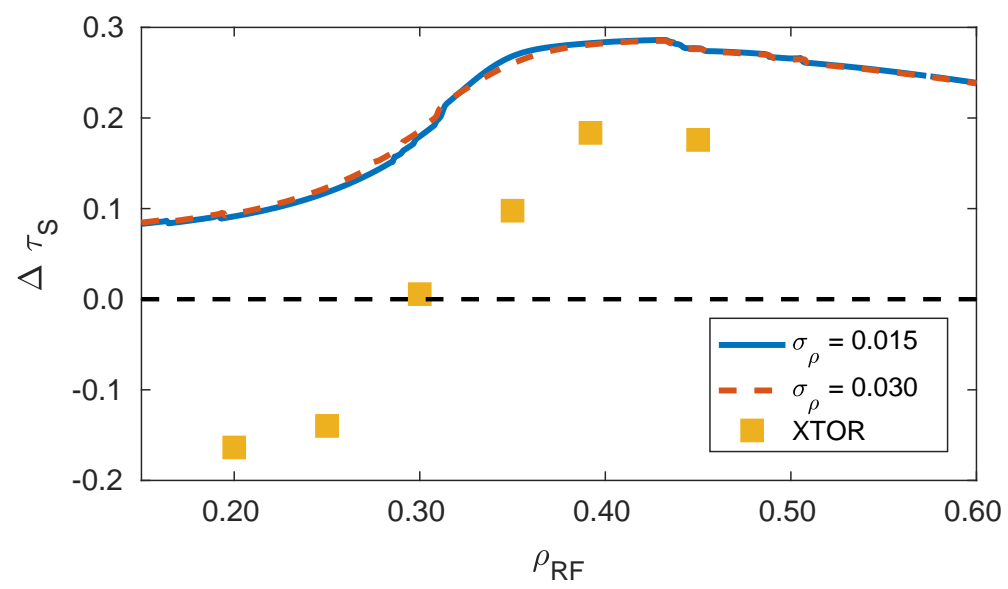

Figure 17. Comparison of the sawtooth periods computed by XTOR and the model developed in equations 14-16 for heating deposition at different values of the radial location $\rho_{R F}$, the injected current being kept constant $\alpha_{C D}=0$. The critical shear used in the model has been set to $s_{1, \text { crit }}=0.19$. The current source $J_{R F}$ is set to 0 .

for the triggering of such modes, as confirmed by experimental observations [14, 65, 15]. In order to illustrate the possible mechanisms behind the triggering of an island by a sawtooth crash, we will refer to a simplified version of the Rutherford equation, which can be used to model the evolution of the normalized island width

$$
0.82 S \frac{\mathrm{d} W}{\mathrm{dt}}=a \Delta^{\prime}+6.35 \bar{J}_{b s} \frac{q}{s} \frac{W}{W^{2}+W_{b s}^{2}}
$$

where $W$ is the island width normalized to the minor radius, $S$ the Lundquist number, $\bar{J}_{b s}=\left(\mu_{0} R_{0} / B_{0}\right) J_{b s}$ is the normalized bootstrap current, $J_{b s}$ is the bootstrap current on the magnetic surface of interest, $q$ is the safety factor, $s$ is the magnetic shear on this 
Non-linear MHD simulations of sawteeth and their control by current and power depositions25

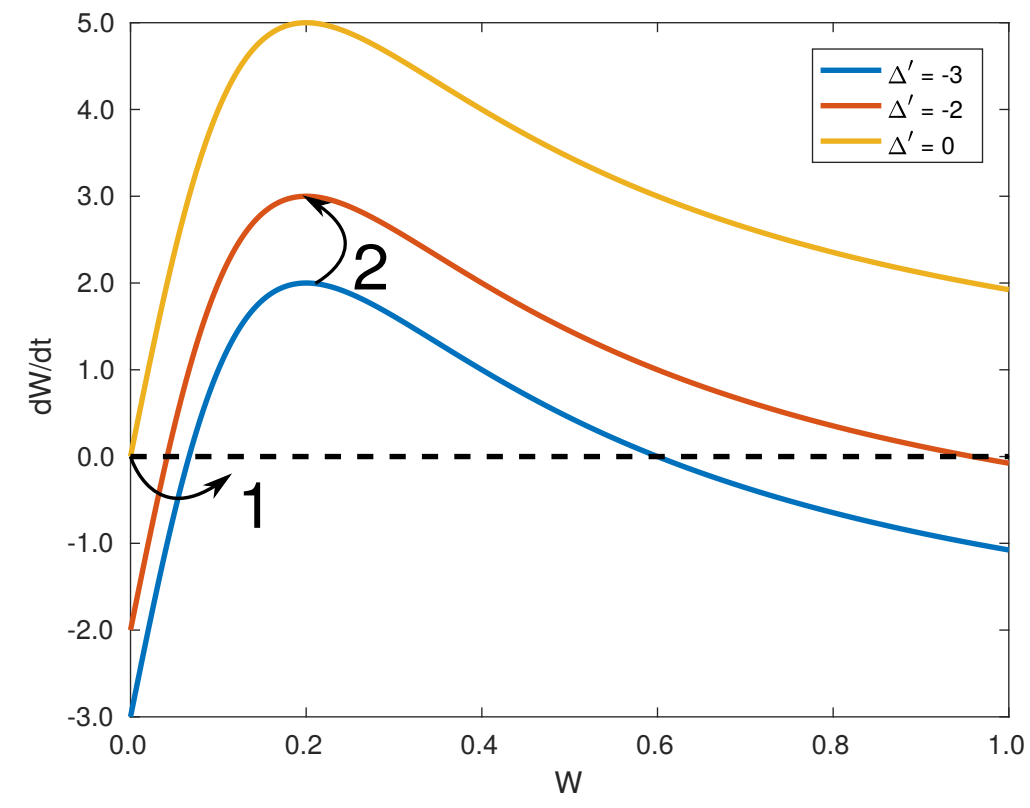

Figure 18. Typical phase diagram of a metastable tearing mode, as described by equation 28. The first mechanism, denoted as "1", refers to the direct seeding of the island by a perturbation (a sawtooth crash, for instance) that directly provide a seed above the critical width. The second mechanism ("2") refers to the linear destabilization of the mode, that leads to a decrease of the critical width and a possible increase of the growth rate. The yellow curve corresponds to an extreme case of mechanism 2 where a strong modification of the $\Delta^{\prime}$ makes the mode linearly unstable. We emphasize that the purpose of this figure is to illustrate the different mechanisms that could explain the onset of an island. Thus the set of parameters chosen to plot is arbitrary, and does not correspond to what we have in the simulations that we present in this paper.

magnetic surface and $W_{b s}$ is a characteristic island width related to the transport inside the island. For simplicity, we have neglected terms such as the curvature stabilization [66], the role of the polarization current or the dependence of $\Delta^{\prime}$ with the island width [67]. Depending on the values of the different parameters, this equation features different equilibrium solutions $(d W / d t=0)$. For illustration purpose, we place ourselves in a typical metastable NTM case, with $\Delta^{\prime}<0$ and $\Delta^{\prime}, q, s$ and $W_{b s}$ such that two equilibrium solutions exist, a stable one, which is the largest one and corresponds to the saturation width of the island, and an unstable one, which corresponds to the critical width. A typical phase diagram associated with equation 28 is plotted in figure 18 . From equation 28 , the critical width $W_{\text {crit }}$ of the island can be written as

$$
W_{\text {crit }}=\frac{-6.35 J_{b s} \frac{q}{s}-\sqrt{\left[6.35 J_{b s} \frac{q}{s}\right]^{2}-4 a^{2}{\Delta^{\prime}}^{2} W_{b s}^{2}}}{2 a \Delta^{\prime}}
$$

From figure 18, two mechanisms can be imagined to trigger an island from a sawtooth crash. The first one relies in the generation, during the crash, of large magnetic perturbations. Because of poloidal and toroidal couplings, some harmonics of the $(1,1)$ mode could be excited and go beyond the critical width of the associated magnetic 
island, thus leading to the triggering of the mode (arrow 1 in figure 18). Another mechanism relies on the modification of the linear stability of the mode (quantified by the $\Delta^{\prime}$ parameter [68]) due to the brutal modification of the current profile induced by the sawtooth [69]. A metastable mode, for which $\Delta^{\prime}<0$, could briefly become less stable (arrow 2 in figure 18), or even unstable $\left(\Delta^{\prime}>0\right.$ ) growing up to a width larger than its critical width, so that even after the relaxation of the current profile to its equilibrium value, the island remains in the plasma. From equation 29, we obtain that if $\Delta^{\prime}$ (defined as negative) increases (that is, gets closer to 0 ) then the island critical width decreases, which is understandable as the linear stability of the island is reduced. This allows us to describe the possible interplay between the two mechanisms. As the sawtooth crash occurs, the nonlinear couplings will generate a seed island. If this seed is larger than the equilibrium critical width of the island, then an island is triggered. However, if it is not the case, the $\Delta^{\prime}$ peak that we observe will transiently reduce the critical width of the island, potentially allowing the island to (transiently) start a non-linear growth phase. Since the $\Delta^{\prime}$ peak is very sudden and short, as shown later in figure 21, the critical width will quickly relax to its equilibrium value. However, if the mode growth rate is high enough so that the mode width remains higher than the critical width as the latter re-increases, then the mode will "survive" after the sawtooth. There is a mechanism of "cooperation-competition" between the two effects. The outcome likely depends on the equilibrium parameters, as well as on the amplitude of both the harmonics and $\Delta^{\prime}$ kicks provided by the sawtooth. In this article we do not include the contribution from the bootstrap current in XTOR's Ohm's law, and thus do not attempt to model the nonlinear seeding of NTMs, which would be computationally expensive. We investigate these two possible mechanisms separately and verify from our simulations that they are indeed present and thus possible candidates for NTMs triggering. We then show how these mechanisms can be controlled by modifying the sawtooth period.

\subsection{Nonlinear destabilization of tearing modes}

We start by investigating the role of nonlinear couplings on the excitation of harmonics that could lead to the onset of a tearing mode. In figure 19 we have plotted the evolutions of $M_{2 / 1}^{0.25}$ and $M_{3 / 2}^{0.25}$ where $M_{2 / 1}$ is the magnetic energy of the $(2,1)$-mode and $M_{3 / 2}$ is the magnetic energy of the $(3,2)$-mode. By definition $M^{0.25}$ is proportional to the island width $W$. From these plots, it is clear that longer sawteeth, associated with stronger crashes, lead to higher -transient- amplitude of the $(2,1)$ and $(3,2)$ modes. Thus, these harmonics are susceptible to get close or even above a possible critical width threshold, which would lead to the onset of the mode and its saturation. The triggering of magnetic islands being a threat for the operation of future tokamaks, the control of this triggering is of interest for fusion research. We therefore study the impact of current or power deposition on the excitation of harmonics. In the center panel of figure 20 the maximum value of $M_{2 / 1}^{0.25}$ and $M_{3 / 2}^{0.25}$ reached during sawtooth activity in the presence of RF current deposition are plotted as a function of the deposition location. Depositions way outside 
Non-linear MHD simulations of sawteeth and their control by current and power depositions 27
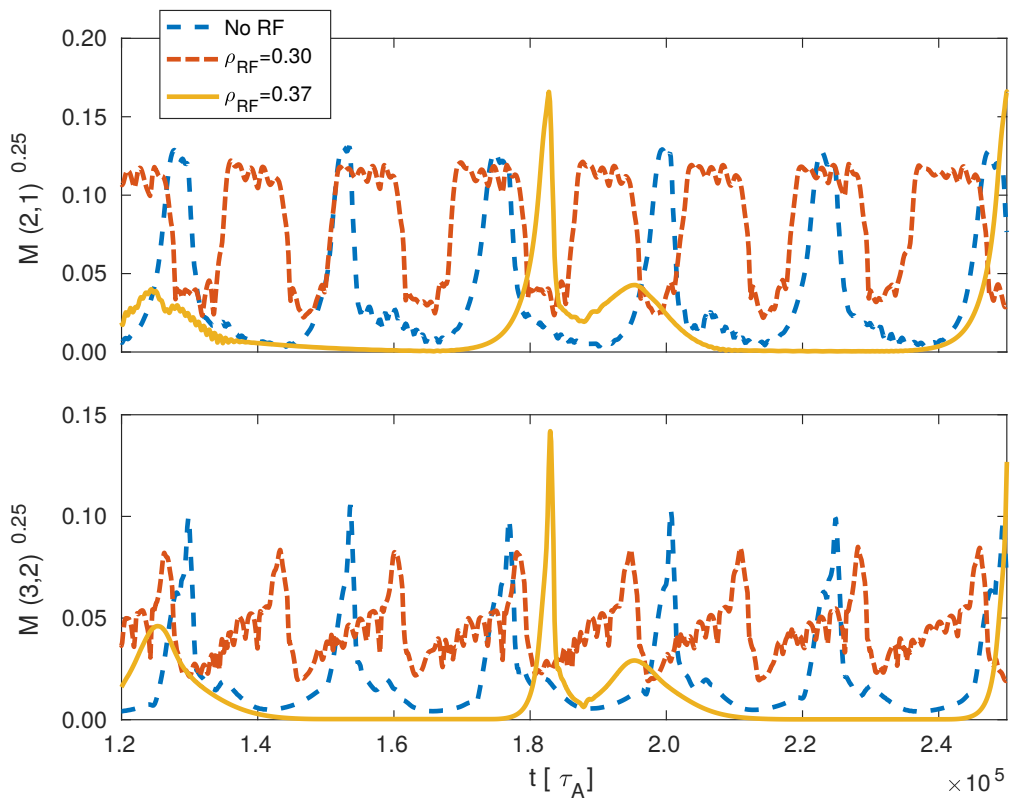

Figure 19. (top) Evolution of $M_{2 / 1}^{0.25}$ where $M_{2 / 1}$ is the magnetic energy of the $(2,1)$ mode. (bottom) Evolution of $M_{3 / 2}^{0.25}$ where $M_{3 / 2}$ is the magnetic energy of the (3,2)mode. In both cases, longer sawtooth periods are correlated to higher peak value and thus to an easier trespassing of a possible critical width threshold. The current deposition is set such that $I_{R F} / I_{P}=1 \%$.

the inversion radius $\left(\rho_{R F}=0.4,0.45\right)$ appear to reduce the maximum amplitude of the perturbation that is reached. Since for these depositions, the sawtooth period is barely modified, we can expect that this modification results from the global change of the $(2,1)$ and $(3,2)$ stability due to the current profile modification. Depositions on the inversion radius tend to increase both the sawtooth period (see figure 7) and the $(2,1)$ and $(3,2)$ perturbations, as they lead to stronger crashes. Depositions inside, but close to the inversion radius appear to be the most efficient to reduce the amplitude reached by the perturbations, although in our case, this reduction is small. Interestingly, depositions further inside the inversion radius, while still reducing the sawtooth period (figure 7 ), can lead to higher excitation of the $(2,1)$ or $(3,2)$ harmonics, likely because of a global modification of the current profile. This indicates that, in the perspective of sawtooth control, there might be a "sweet spot", right inside the inversion radius (in our case, between $\rho_{R F}=0.3$ and 0.33 ) where the current deposition is efficient for both sawtooth period reduction and mode coupling reduction. Outside this region, one might still have a beneficial effect on one of these aspects, but maybe not both. Finally, in the bottom panel of figure 20, we have plotted the evolution of the maximum mixing radius reached during sawteeth in presence of current deposition, whose position is compared to the position of $q=3 / 2$ and $q=2$. We find that a higher peak mixing radius is correlated with a higher destabilization of the mode, which is compatible with experimental observations [69]. 

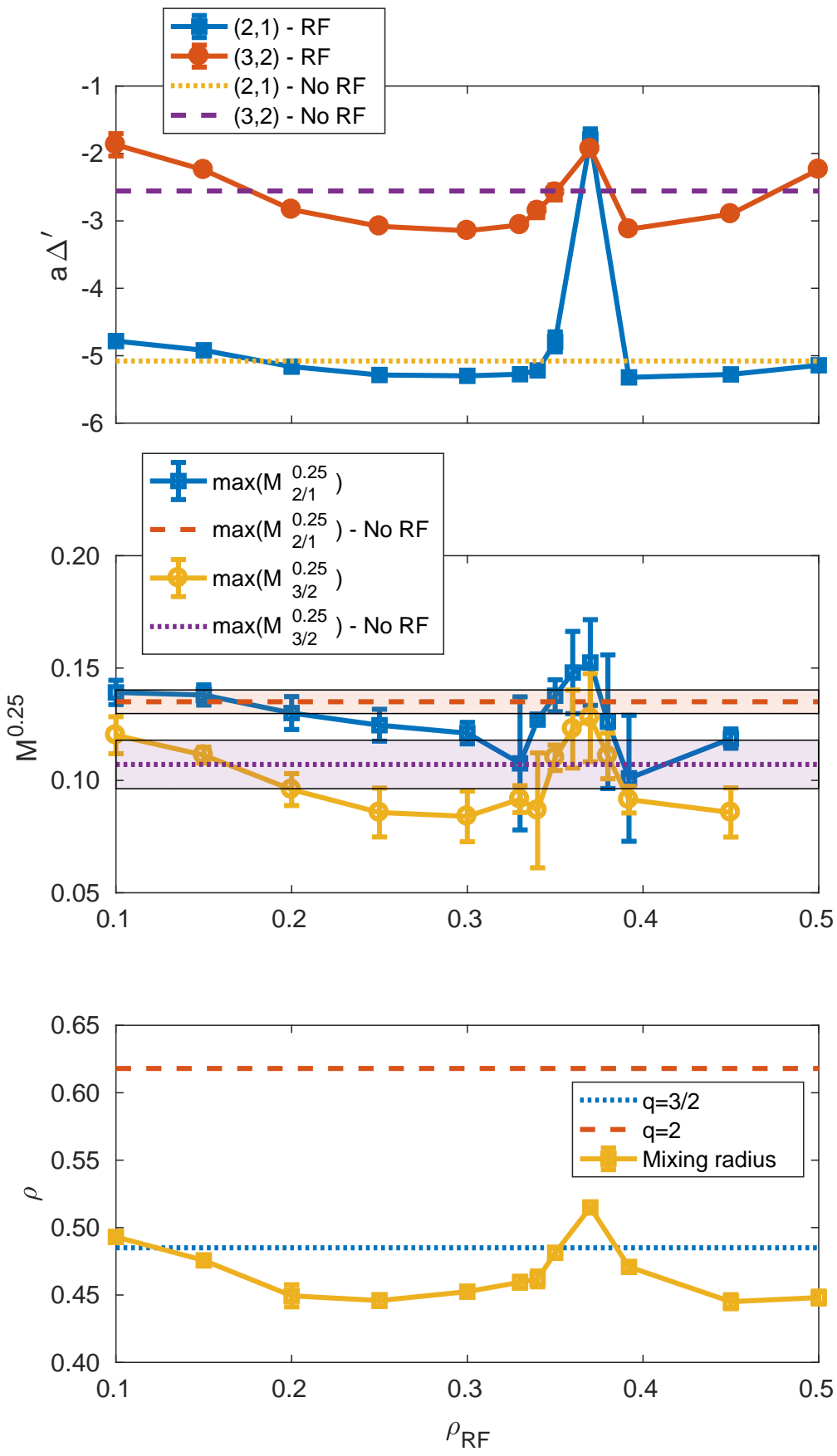

Figure 20. (top) Maximum value of $a \Delta_{2,1}^{\prime}$ and $a \Delta_{3,2}^{\prime}$ reached during sawtooth activity in the presence of RF current deposition $\left(I_{R F} / I_{P}=1 \%\right)$ as a function of the deposition location. The dashed and dotted lines indicate the maximum amplitudes of $a \Delta_{2,1}^{\prime}$ and $a \Delta_{3,2}^{\prime}$ reached in the absence of RF current deposition. (middle) Maximum value of $M_{2 / 1}^{0.25}$ and $M_{3 / 2}^{0.25}$ reached during sawtooth activity in the presence of $\mathrm{RF}$ current deposition $\left(I_{R F} / I_{P}=1 \%\right)$ as a function of the deposition location. The dashed and dotted lines indicate the maximum amplitudes of $M_{2 / 1}^{0.25}$ and $M_{3 / 2}^{0.25}$ reached in the absence of RF current deposition. (bottom) Maximum value of the mixing radius reached during sawtooth activity in the presence of RF current deposition $\left(I_{R F} / I_{P}=1 \%\right)$ as a function of the deposition location, compared to the position of $q=3 / 2$ and $q=2$. 


\subsection{Linear stability evolution during crashes}

We now investigate the modification of the mode linear stability (quantified by the stability index $\Delta^{\prime}$ ) during a sawtooth crash. To do so, the q-profile for many time slices during a sawtooth crash is reconstructed using the $n=0$ components of the magnetic field. The cylindrical $\Delta^{\prime}$ is then evaluated for a given $(m, n)$-tearing mode for each time slice. This is represented in figure 21, where we have plotted the evolution of the $\Delta^{\prime}$ parameters for the $(2,1)$ and $(3,2)$ tearing modes in the absence of current or power depositions. As one can see, the sawtooth crash induces a sudden increase of $\Delta^{\prime}$. The $\Delta^{\prime}$ peak remains however very limited in time, and thus it is not clear if the associated decrease of mode linear stability and reduction of the critical width would be sufficient to let a mode develop. Investigating this more in detail would require nonlinear simulations of the island triggering, which is beyond the scope of this paper. Following the approach of the previous sections, we have plotted in figure 21 the evolution of the $\Delta^{\prime}$ parameter for the $(2,1)$ and $(3,2)$ modes for cases where localized current deposition is used to modify the sawtooth period. For both modes, we see that in the presence of a RF current deposition, the evolution of $\Delta^{\prime}$ is indeed modified. In the case of a deposition at $\rho_{R F}=0.30$, the $\Delta^{\prime}$ is generally smaller (hence the mode is more linearly stable), while a deposition outside the inversion radius leads to higher peak value of the $\Delta^{\prime}$. However, the peak is followed by a rather long period during which the $\Delta^{\prime}$ is consistently smaller than in other cases, which could help to recover from a possible

triggering, by increasing the linear stability of the mode. In the top panel of figure 20, we have plotted the peak of the $\Delta^{\prime}$ as a function of the deposition location, which shows that depositions inside the sawtooth inversion radius also lead to lower $\Delta^{\prime}$ modifications. On the contrary, increasing the sawtooth period leads to stronger crashes (stronger increases of $\Delta^{\prime}$ ), which are thus more susceptible to trigger additional modes. This is consistent with experimental observations [15].

\section{Conclusion}

In this article, we have investigated the influence of current or power deposition on the dynamics of sawteeth. A proper formulation of the current source present in the Ohm's law allowed us to simulate fully non-inductive plasma current and mostly inductiveplasma current scenarios, while keeping the freedom to define a resistivity varying according to the Spitzer's law.

We have shown that depending on the intensity and the location of the current that is deposited, the frequency of the sawteeth can be controlled. With co-current injection, a deposition inside the inversion radius tends to reduce the sawtooth period, while a deposition outside tends to increase it, in agreement with experimental observations. With counter-current depositions, the opposite is observed, and depositions inside the inversion radius lead to an increase of the sawtooth period. We have shown that the results for power depositions are somewhat similar to the ones for current depositions, 
Non-linear MHD simulations of sawteeth and their control by current and power depositions30
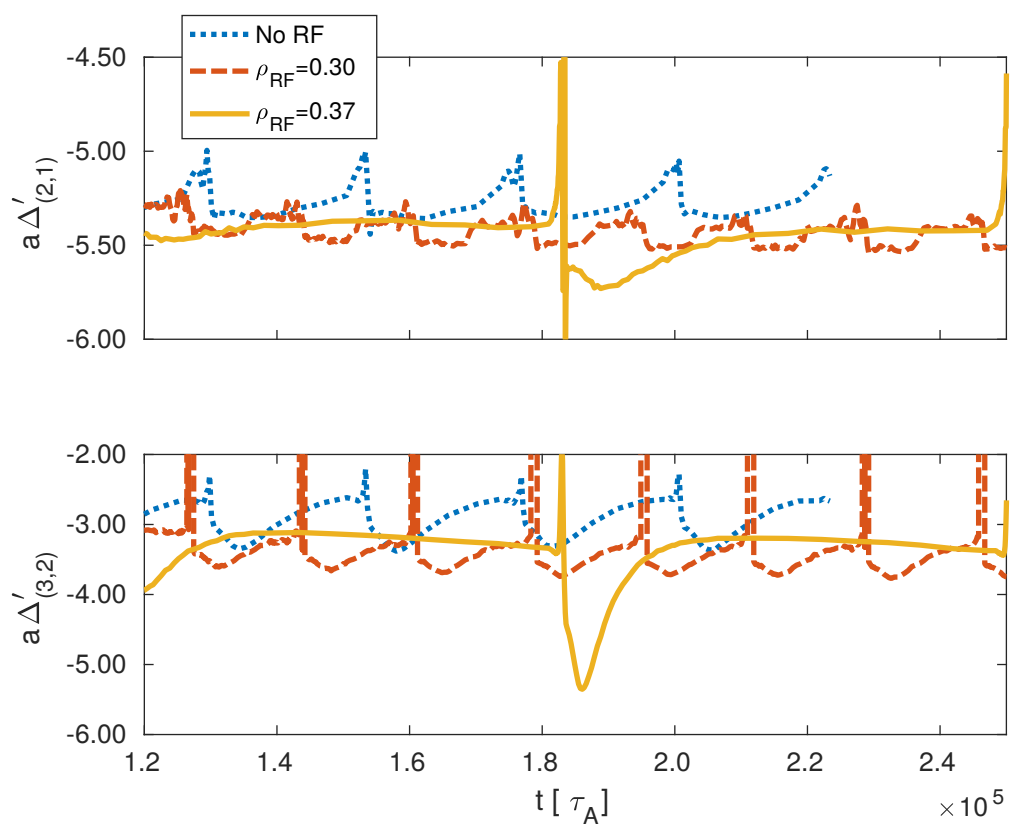

Figure 21. (top) Evolution of $a \Delta_{(2,1)}^{\prime}$ where $\Delta_{(2,1)}^{\prime}$ is the stability index for the $(2,1)$ mode, in the presence of current deposition by ECCD-like source term. For readability of the figure, the peak value at $\rho_{E C}=0.37(\approx-1.6)$ is out of the scale. (bottom) Evolution of $a \Delta_{(3,2)}^{\prime}$ where $\Delta_{(3,2)}^{\prime}$ is the stability index for the $(3,2)$-mode, in the presence of current deposition by ECCD-like source term. Longer sawtooth period are associated to higher peak value and thus to an easier possible destabilization. The current deposition is set such that $I_{R F} / I_{P}=1 \%$.

even though the dynamics at play is more complex, due, in part, to the modification of the diamagnetic effects caused by the change in the pressure profile, which affects more in depth the stability of the internal kink mode. In the case of current deposition, we have shown that the observed behavior is consistent with a critical-shear model, the current deposition impacting the sawtooth period by altering the dynamics at which the shear on the $q=1$ surface reaches its critical value. This is in good agreement with theoretical works $[32,33]$. However, our simulations go beyond these analytical models by the full self-consistent description, in the MHD framework, of the dynamics of the current and of the pressure. They show in particular a mismatch with the analytical work in the case of power depositions, where at the largest deposited power, XTOR simulations show a different trend than the models. The simulations also reveals that the dynamics of the shear between the crash is complex, as shown in figure 8 , and thus cannot be fully understood using a threshold model with an instantaneous modification of the profiles at the crashes and a pure current diffusion in between.

We then studied the impact of the ohmic current amplitude on the sawtooth dynamics. We have shown that this alters slightly the sawtooth dynamics, and that it constraints the control method that can be used to modify the sawtooth period. In particular, in the case of fully non-inductive plasma, a power deposition has a much less pronounced impact on the shear (in the absence of bootstrap current), and thus mostly 
plays a role through the modification of diamagnetic effects. It should be noted that in this article, we took the stance to investigate the effects of local heating and local currentdrive separately, in order to evaluate the mechanisms at play and quantify the impact of each one. In experiments however, the situation is more intricate, as current driven by ECCD generally comes with a fraction of local heating. Thus, both effects are entangled, which should be considered for the analysis. The interplay between the two is however beyond the scope of this paper. We reported observations of the modification of the sawtooth shape by both current and power depositions, in agreement with experimental observations. While we did not provide an explanation for this, which is left for future work, we suppose that a likely player is the modification of the internal kink mode growth rate and stability threshold due to the modification of the current profiles.

In the last section of this paper, we investigated the triggering of secondary instabilities (tearing modes) by the sawtooth crashes. While we did not simulate the full nonlinear triggering of a magnetic island, we were able to evidence that the sawtooth crash leads to a brutal decrease of the tearing modes stability (through an increase of the $\Delta^{\prime}$ parameter). If $\Delta^{\prime}$ were to become greater than zero, then some modes could become temporary unstable and might grow below a critical width that would allow them to remain in their metastable branch. We also evidenced that the poloidal and toroidal couplings can lead to relatively large amplitude of different harmonics, that again might become large enough to allow for a mode to go above a marginal width and start to grow up to a metastable critical width. We then showed that by depositing current inside the sawtooth inversion radius, and thus by increasing their frequency, these effects could be reduced.

To summarize, we have shown from first-principle MHD simulations the impact of current and power depositions on the sawtooth dynamics, and we have confirmed the existence of a critical shear determining the onset of the sawtooth crash. The dynamics of the magnetic shear at $q=1$ in between two crashes is found to be instrumental in the core temperature evolution. It however shows in many situations a complex behavior that cannot be captured by simple redistribution rules of complete [4] or incomplete [33] reconnection occurring at the sawtooth onset time. The pressure dynamics reflects this complex evolution and shows a large variety of sawtooth shapes that is also observed experimentally. We further have shown how sawtooth control can be used to prevent the onset of additional instability, as already evidenced in experiments and as predicted by simpler models.

\section{Acknowledgments}

This work was supported in part by the Swiss National Science Foundation. This work is part of the AMICI project (ANR-14-CE32-0004-01) funded by the Agence Nationale pour la Recherche. Numerical resources were provided by CINES and TGCC of GENCI (x2015056348), Mésocentre of Aix-Marseille University (b009) and Helios of IFERC-CSC (MaCoToP). This work has been carried out within the frameworks of 
the French Research Federation for Magnetically Confined Fusion (FR-FCM) and of the EUROfusion Consortium and has received funding from the Euratom research and training programme 2014-2018 under grant agreement No 633053 for the project WP17ENR-CEA-06. The views and opinions expressed herein do not necessarily reflect those of the European Commission.

\section{References}

[1] Hinrich Lütjens and Jean-François Luciani. XTOR-2F: A fully implicit Newton-Krylov solver applied to nonlinear 3D extended MHD in tokamaks. Journal of Computational Physics, 229(21):8130 - 8143, 2010.

[2] S. von Goeler, W. Stodiek, and N. Sauthoff. Studies of Internal Disruptions and $m=1$ Oscillations in Tokamak Discharges with Soft X-Ray Techniques. Phys. Rev. Lett., 33:1201-1203, Nov 1974.

[3] M. N. Bussac, R. Pellat, D. Edery, and J. L. Soule. Internal kink modes in toroidal plasmas with circular cross sections. Phys. Rev. Lett., 35:1638-1641, Dec 1975.

[4] B.B. Kadomtsev. Soviet Journal of Plasma Physics, 1:389, 1975.

[5] R. J. Hastie. Sawtooth instability in tokamak plasmas. Astrophysics and Space Science, 256(1):177-204, 1997.

[6] R Sabot, F Clairet, G D Conway, L Cupido, X Garbet, G Falchetto, T Gerbaud, S Hacquin, P Hennequin, S Heuraux, C Honore, G Leclert, L Meneses, A Sirinelli, L Vermare, and A Truc. Recent results on turbulence and MHD activity achieved by reflectometry. Plasma Physics and Controlled Fusion, 48(12B):B421, 2006.

[7] A. W. Edwards, D. J. Campbell, W. W. Engelhardt, H. U. Fahrbach, R. D. Gill, R. S. Granetz, S. Tsuji, B. J. D. Tubbing, A. Weller, J. Wesson, and D. Zasche. Rapid Collapse of a Plasma Sawtooth Oscillation in the JET Tokamak. Phys. Rev. Lett., 57:210-213, Jul 1986.

[8] M. Yamada, F. M. Levinton, N. Pomphrey, R. Budny, J. Manickam, and Y. Nagayama. Investigation of magnetic reconnection during a sawtooth crash in a hightemperature tokamak plasma. Physics of Plasmas, 1(10):3269-3276, 1994.

[9] D. Vezinet, V. Igochine, M. Weiland, Q. Yu, A. Gude, D. Meshcheriakov, M. Sertoli, the Asdex Upgrade Team, and the EUROfusion MST1 Team. Non-monotonic growth rates of sawtooth precursors evidenced with a new method on asdex upgrade. Nuclear Fusion, 56(8):086001, 2016.

[10] R. Dux, A.G. Peeters, A. Gude, A. Kallenbach, R. Neu, and ASDEX Upgrade Team. Z dependence of the core impurity transport in ASDEX Upgrade $\mathrm{H}$ mode discharges. Nuclear Fusion, 39(11):1509, 1999.

[11] M.F.F. Nave, J. Rapp, T. Bolzonella, R. Dux, M.J. Mantsinen, R. Budny, P. Dumortier, M. von Hellermann, S. Jachmich, H.R. Koslowski, G. Maddison, A. Messiaen, P. Monier-Garbet, J. Ongena, M.E. Puiatti, J. Strachan, G. Telesca, B. Unterberg, M. Valisa, P. de Vries, and contributors to the JET-EFDA Workprogramme. Role of sawtooth in avoiding impurity accumulation and maintaining good confinement in JET radiative mantle discharges. Nuclear Fusion, 43(10):1204, 2003.

[12] T. Nakano, N. Asakura, H. Kubo, J. Yanagibayashi, and Y. Ueda. Tungsten accumulation in H-mode plasmas of JT-60U. Nuclear Fusion, 49(11):115024, 2009.

[13] M. Sertoli, T. Odstrcil, C. Angioni, and ASDEX Upgrade Team. Interplay between central ECRH and saturated $(\mathrm{m}, \mathrm{n})=(1,1)$ MHD activity in mitigating tungsten accumulation at ASDEX Upgrade. Nuclear Fusion, 55(11):113029, 2015.

[14] O. Sauter, E. Westerhof, M. L. Mayoral, B. Alper, P. A. Belo, R. J. Buttery, A. Gondhalekar, T. Hellsten, T. C. Hender, D. F. Howell, T. Johnson, P. Lamalle, M. J. Mantsinen, F. Milani, M. F. F. Nave, F. Nguyen, A. L. Pecquet, S. D. Pinches, S. Podda, and J. Rapp. Control of Neoclassical Tearing Modes by Sawtooth Control. Phys. Rev. Lett., 88(10):105001, Feb 2002.

[15] R.J. Buttery, T.C. Hender, D.F. Howell, R.J. La Haye, S. Parris, O. Sauter, C.G. Windsor, and 
Non-linear MHD simulations of sawteeth and their control by current and power depositions33

JET-EFDA Contributors. On the form of NTM onset scalings. Nuclear Fusion, 44(5):678-685, 2004.

[16] I.T. Chapman, R.J. Buttery, S. Coda, S. Gerhardt, J.P. Graves, D.F. Howell, A. Isayama, R.J. La Haye, Y. Liu, P. Maget, M. Maraschek, S. Sabbagh, O. Sauter, the ASDEX Upgrade, DIIID, HL-2A, JT-60U, MAST, NSTX, TCV, Tore Supra Teams, and JET-EFDA Contributors. Empirical scaling of sawtooth period for onset of neoclassical tearing modes. Nuclear Fusion, 50(10):102001, 2010.

[17] G.P. Canal, B.P. Duval, F. Felici, T.P. Goodman, J.P. Graves, A. Pochelon, H. Reimerdes, O. Sauter, D. Testa, and the TCV Team. Fast seeding of NTMs by sawtooth crashes in TCV and their preemption using ECRH. Nuclear Fusion, 53(11):113026, 2013.

[18] V. Igochine, A. Gude, S. Günter, K. Lackner, Q. Yu, L. Barrera Orte, A. Bogomolov, I. Classen, R. M. McDermott, and N. C. Luhmann Jr. Conversion of the dominantly ideal perturbations into a tearing mode after a sawtooth crash. Physics of Plasmas, 21(11):110702, 2014.

[19] V. Igochine, I. Classen, M. Dunne, A. Gude, S. Günter, K. Lackner, R.M. McDermott, M. Sertoli, D. Vezinet, M. Willensdorfer, Q. Yu, H. Zohm, and ASDEX Upgrade Team. Tearing mode formation induced by internal crash events at different betaN. Nuclear Fusion, 57(3):036015, 2017.

[20] M.F.F. Nave and J.A. Wesson. Mode locking in tokamaks. Nuclear Fusion, 30(12):2575, 1990.

[21] J. Wesson. Tokamaks. Oxford Science Publications, 1997.

[22] F Porcelli. Fast particle stabilisation. Plasma Physics and Controlled Fusion, 33(13):1601, 1991.

[23] Jonathan P. Graves. Internal kink mode stabilization and the properties of auxiliary heated ions. Physics of Plasmas, 12(9):090908, 2005.

[24] I T Chapman. Controlling sawtooth oscillations in tokamak plasmas. Plasma Physics and Controlled Fusion, 53(1):013001, 2011.

[25] R. B. White, P. H. Rutherford, P. Colestock, and M. N. Bussac. Sawtooth stabilization by energetic trapped particles. Phys. Rev. Lett., 60:2038-2041, May 1988.

[26] Jonathan P. Graves. Influence of asymmetric energetic ion distributions on sawtooth stabilization. Phys. Rev. Lett., 92:185003, May 2004.

[27] I T Chapman, J P Graves, T Johnson, O Asunta, P Bonoli, M Choi, E F Jaeger, M Jucker, and O Sauter. Sawtooth control in ITER using ion cyclotron resonance heating. Plasma Physics and Controlled Fusion, 53(12):124003, 2011.

[28] M. F. F. Nave, H. R. Koslowski, S. Coda, J. Graves, M. Brix, R. Buttery, C. Challis, C. Giroud, M. Stamp, and P. de Vries. Exploring a small sawtooth regime in joint european torus plasmas with counterinjected neutral beams. Physics of Plasmas, 13(1):014503, 2006.

[29] I T Chapman, I Jenkins, R V Budny, J P Graves, S D Pinches, S Saarelma, and JET EFDA Contributors. Sawtooth control using off-axis nbi. Plasma Physics and Controlled Fusion, 50(4):045006, 2008.

[30] I.T. Chapman, S.D. Pinches, H.R. Koslowski, Y. Liang, A. Krmer-Flecken, the TEXTOR Team, and M. de Bock. Sawtooth stability in neutral beam heated plasmas in textor. Nuclear Fusion, 48(3):035004, 2008.

[31] J P Graves, M Lennholm, I T Chapman, E Lerche, M Reich, B Alper, V Bobkov, R Dumont, J M Faustin, P Jacquet, F Jaulmes, T Johnson, D L Keeling, Yueqiang Liu, T Nicolas, S Tholerus, T Blackman, I S Carvalho, R Coelho, D Van Eester, R Felton, M Goniche, V Kiptily, I Monakhov, M F F Nave, C Perez von Thun, R Sabot, C Sozzi, and M Tsalas. Sawtooth control in JET with ITER relevant low field side resonance ion cyclotron resonance heating and ITERlike wall. Plasma Physics and Controlled Fusion, 57(1):014033, 2015.

[32] L. Zakharov, B. Rogers, and S. Migliuolo. The theory of the early nonlinear stage of $\mathrm{m}=1$ reconnection in tokamaks*. Physics of Fluids B: Plasma Physics, 5(7):2498-2505, 1993.

[33] F Porcelli, D Boucher, and M N Rosenbluth. Model for the sawtooth period and amplitude. Plasma Physics and Controlled Fusion, 38(12):2163, 1996.

[34] V.P. Bhatnagar, D.F.H. Start, J. Jacquinot, F. Chaland, A. Cherubini, and F. Porcelli. Local 
Non-linear MHD simulations of sawteeth and their control by current and power depositions34

magnetic shear control in a tokamak via fast wave minority ion current drive: theory and experiments in jet. Nuclear Fusion, 34(12):1579, 1994.

[35] Z.A. Pietrzyk, A. Pochelon, T.P. Goodman, M.A. Henderson, J.-P. Hogge, H. Reimerdes, M.Q. Tran, R. Behn, I. Furno, J.-M. Moret, C. Nieswand, J. Rommers, O. Sauter, W. van Toledo, H. Weisen, F. Porcelli, and K.A. Razumova. Behaviour of central plasma relaxation oscillations during localized electron cyclotron heating on the TCV tokamak. Nuclear Fusion, 39(5):587, 1999.

[36] Y. Ikeda, S. Ide, T. Suzuki, A. Kasugai, K. Takahashi, K. Kajiwara, A. Isayama, T. Oikawa, K. Hamamatsu, Y. Kamada, T. Fujita, K. Sakamoto, S. Moriyama, M. Seki, R. Yoshino, T. Imai, K. Ushigusa, T. Fujii, and JT-60 Team. Ecrf experiments for local heating and current drive by fundamental o-mode launch from the low-field side on jt-60u. Nuclear Fusion, 42(4):375, 2002.

[37] C. Angioni, T.P. Goodman, M.A. Henderson, and O. Sauter. Effects of localized electron heating and current drive on the sawtooth period. Nuclear Fusion, 43(6):455, 2003.

[38] L.-G. Eriksson, A. Mueck, O. Sauter, S. Coda, M. J. Mantsinen, M.-L. Mayoral, E. Westerhof, R. J. Buttery, D. McDonald, T. Johnson, J.-M. Noterdaeme, and P. de Vries. Destabilization of fast-ion-induced long sawteeth by localized current drive in the jet tokamak. Phys. Rev. Lett., 92:235004, Jun 2004.

[39] H. Zohm, G. Gantenbein, F. Leuterer, A. Manini, M. Maraschek, Q. Yu, and the ASDEX Upgrade Team. Control of MHD instabilities by ECCD: ASDEX Upgrade results and implications for ITER. Nuclear Fusion, 47(3):228, 2007.

[40] M. Lennholm, L.-G. Eriksson, F Turco, F. Bouquey, C. Darbos, R. Dumont, G. Giruzzi, M. Jung, R. Lambert, R. Magne, D. Molina, P. Moreau, F. Rimini, J-L. Segui, S. Song, and E. Traisnel. Demonstration of Effective Control of Fast-Ion-Stabilized Sawteeth by Electron-Cyclotron Current Drive. Phys. Rev. Lett., 102:115004, Mar 2009.

[41] I.T. Chapman, R.J. La Haye, R.J. Buttery, W.W. Heidbrink, G.L. Jackson, C.M. Muscatello, C.C. Petty, R.I. Pinsker, B.J. Tobias, and F. Turco. Sawtooth control using electron cyclotron current drive in ITER demonstration plasmas in DIII-D. Nuclear Fusion, 52(6):063006, 2012.

[42] I T Chapman, V Igochine, M Maraschek, P J McCarthy, G Tardini, the ASDEX Upgrade ECRH Group, and the ASDEX Upgrade Team. Sawtooth control using electron cyclotron current drive in the presence of energetic particles in high performance ASDEX Upgrade plasmas. Plasma Physics and Controlled Fusion, 55(6):065009, 2013.

[43] I. Furno, C. Angioni, F. Porcelli, H. Weisen, R. Behn, T.P. Goodman, M.A. Henderson, Z.A. Pietrzyk, A. Pochelon, H. Reimerdes, and E. Rossi. Understanding sawtooth activity during intense electron cyclotron heating experiments on TCV. Nuclear Fusion, 41(4):403, 2001.

[44] E. Westerhof, J.A. Hoekzema, G.M.D. Hogeweij, R.J.E. Jaspers, F.C. Schüller, C.J. Barth, W.A. Bongers, A.J.H. Donn, P. Dumortier, A.F. van der Grift, J.C. van Gorkom, D. Kalupin, H.R. Koslowski, A. Krmer-Flecken, O.G. Kruijt, N.J. Lopes Cardozo, P. Mantica, H.J. van der Meiden, A. Merkulov, A. Messiaen, J.W. Oosterbeek, T. Oyevaar, A.J. Poelman, R.W. Polman, P.R. Prins, J. Scholten, A.B. Sterk, C.J. Tito, V.S. Udintsev, B. Unterberg, M. Vervier, G. van Wassenhove, and TEC Team. Electron cyclotron resonance heating on TEXTOR. Nuclear Fusion, 43(11):1371, 2003.

[45] Yi Yuan, Liqun Hu, Liqing Xu, Xiaoguang Wang, Xiaojie Wang, Handong Xu, Zhengping Luo, Kaiyun Chen, Shiyao Lin, Yanmin Duan, Pengxiang Chang, Hailin Zhao, Kaiyang He, and Yunfeng Liang. Control of sawtooth via ECRH on EAST tokamak. Physics of Plasmas, 23(6):062503, 2016.

[46] M. Lauret, F. Felici, G. Witvoet, T.P. Goodman, G. Vandersteen, O. Sauter, M.R. de Baar, and the TCV team. Demonstration of sawtooth period locking with power modulation in TCV plasmas. Nuclear Fusion, 52(6):062002, 2012.

[47] T. P. Goodman, F. Felici, O. Sauter, and J. P. Graves. Sawtooth Pacing by Real-Time Auxiliary Power Control in a Tokamak Plasma. Phys. Rev. Lett., 106:245002, Jun 2011.

[48] D. Kim, T. P. Goodman, and O. Sauter. Real-time sawtooth control and neoclassical tearing 
Non-linear MHD simulations of sawteeth and their control by current and power depositions35

mode preemption in ITER. Physics of Plasmas (1994-present), 21(6):061503, 2014.

[49] Alain Marx and Hinrich Lütjens. Hybrid parallelization of the XTOR-2F code for the simulation of two-fluid MHD instabilities in tokamaks. Computer Physics Communications, pages -, 2016.

[50] O. Février, P. Maget, H. Lütjens, J. F. Luciani, J. Decker, G. Giruzzi, M. Reich, P. Beyer, E. Lazzaro, S. Nowak, and the ASDEX Upgrade team. First principles fluid modelling of magnetic island stabilization by electron cyclotron current drive (ECCD). Plasma Physics and Controlled Fusion, 58(4):045015, 2016.

[51] J.-H. Ahn, X. Garbet, H. Lütjens, A. Marx, T. Nicolas, R. Sabot, J.-F. Luciani, R. Guirlet, O. Février, and P. Maget. Non-linear dynamics of compound sawteeth in tokamaks. Physics of Plasmas, 23(5):052509, 2016.

[52] Antoine Merle, Joan Decker, Xavier Garbet, Roland Sabot, Zwinglio Guimares-Filho, and Timothe Nicolas. Stability of the electron-driven fishbone mode. Physics of Plasmas, 19(7):072504, 2012.

[53] Z O Guimaraes-Filho, D Elbeze, R Sabot, D Molina, J-L Segui, C Nguyen, J Decker, P Maget, A Merle, X Garbet, N Dubuit, and S Benkadda. Energetic particle driven magnetohydrodynamic instabilities during relaxation cycles in Tore Supra. Plasma Physics and Controlled Fusion, 53(7):074012, 2011.

[54] H. Lütjens, A. Bondeson, and O. Sauter. The CHEASE code for toroidal MHD equilibria. Computer Physics Communications, 97(3):219-260, 1996.

[55] F D Halpern, D Leblond, H Lütjens, and J-F Luciani. Oscillation regimes of the internal kink mode in tokamak plasmas. Plasma Physics and Controlled Fusion, 53(1):015011, 2011.

[56] Federico D. Halpern, Hinrich Lütjens, and Jean-Francois Luciani. Diamagnetic thresholds for sawtooth cycling in tokamak plasmas. Physics of Plasmas, 18(10):102501, 2011.

[57] F. M. Levinton, L. Zakharov, S. H. Batha, J. Manickam, and M. C. Zarnstorff. Stabilization and onset of sawteeth in tftr. Phys. Rev. Lett., 72:2895-2898, May 1994.

[58] D. Wróblewski and R. T. Snider. Evidence of the complete magnetic reconnection during a sawtooth collapse in a tokamak. Phys. Rev. Lett., 71:859-862, Aug 1993.

[59] Y.B. Nam, J.S. Ko, G.H. Choe, Y. Bae, M.J. Choi, W. Lee, G.S. Yun, S. Jardin, and H.K. Park. Validation of the 'full reconnection model' of the sawtooth instability in kstar. Nuclear Fusion, 58(6):066009, 2018.

[60] H. Soltwisch. Measurement of current-density changes during sawtooth activity in a tokamak by far-infrared polarimetry. Review of Scientific Instruments, 59(8):1599-1604, 1988.

[61] Andreas Letsch, Hartmut Zohm, Francois Ryter, Wolfgang Suttrop, Anja Gude, Franco Porcelli, Clemente Angioni, and Ivo Furno. Incomplete reconnection in sawtooth crashes in asdex upgrade. Nuclear Fusion, 42(9):1055, 2002.

[62] R. T. Snider, D. Content, R. James, John Lohr, M. A. Mahdavi, R. Prater, and B. Stallard. Modification of sawteeth by second harmonic electroncyclotron heating in a tokamak. Physics of Fluids B: Plasma Physics, 1(2):404-413, 1989.

[63] G. Witvoet, M. Steinbuch, M.R. de Baar, N.J. Doelman, and E. Westerhof. Sawtooth period control strategies and designs for improved performance. Nuclear Fusion, 52(7):074005, 2012.

[64] Assyr Abdulle. Explicit Stabilized Runge-Kutta Methods, pages 460-468. Springer Berlin Heidelberg, Berlin, Heidelberg, 2015.

[65] E. Westerhof, O. Sauter, M.L. Mayoral, D.F. Howell, M.J. Mantsinen, M.F.F. Nave, B. Alper, C. Angioni, P. Belo, R.J. Buttery, A. Gondhalekar, T. Hellsten, T.C. Hender, T. Johnson, P. Lamalle, M.E. Maraschek, K.G. McClements, F. Nguyen, A.L. Pecquet, S. Podda, J. Rapp, S.E. Sharapov, M. Zabiego, and contributors to the EFDA JET Workprogramme. Control of sawteeth and triggering of NTMs with ion cyclotron resonance frequency waves in JET. Nuclear Fusion, 42(11):1324-1334, 2002.

[66] Hinrich Lütjens, Jean-François Luciani, and Xavier Garbet. Curvature effects on the dynamics of tearing modes in tokamaks. Physics of Plasmas, 8(10):4267-4270, 2001.

[67] D. F. Escande and M. Ottaviani. Simple and rigorous solution for the nonlinear tearing mode. Physics Letters A, 323(3-4):278 - 284, 2004. 
[68] H. P. Furth, P. H. Rutherford, and H. Selberg. Tearing mode in the cylindrical tokamak. Physics of Fluids, 16(7):1054-1063, 1973.

[69] Patrick Maget, Jean-François Artaud, Lars-Goran Eriksson, Guido Huysmans, A. Lazaros, Philippe Moreau, Maurizio Ottaviani, Jean-Luc Ségui, and Wolfgang Zwingmann. MHD activity triggered by monster sawtooth crashes on Tore Supra. Plasma Physics and Controlled Fusion, 47:357-377, 2005. 